\title{
ULF waves in the outer magnetosphere: Geotail observation 1 transverse waves
}

\author{
Susumu Kokubun \\ Formerly, Solar-Terrestrial Environment Laboratory, Nagoya University, Furo-cho, Chikusa-ku, Nagoya 447-8507, Japan
}

(Received April 2, 2012; Revised December 21, 2012; Accepted December 24, 2012; Online published June 10, 2013)

\begin{abstract}
A survey of ULF waves in the Pc 5 frequency range during the six year period (1995-2000) has been made for the field line resonance waves in the outer magnetosphere, using plasma flow and magnetic field measurements with the Geotail spacecraft. On the morning side a series of wave trains often appears during several hours. In contrast such a series of wave trains with a long duration is rarely observed in the afternoon to evening sector. Most of waves in the afternoon sector are isolated, of which duration is approximately one hour. The existence of a set of preferential frequencies and the pronounced dawn-dusk asymmetry of wave occurrence and wave features, which were found in ground-based and ionospheric measurements of geomagnetic ULF pulsations, were statistically confirmed. It is also noted that the background plasma flow is sunward in the evening sector without exception. Transverse waves are generally observed in the condition of plasma $\beta$ below 1.5. High $\beta$ cases are mostly associated with events on the dusk side. As for the relation to solar wind conditions, Pc 5 waves tend to occur under the condition of more radial than the average IMF spiral angle, or of low cone angle.
\end{abstract}

Key words: Pc 5 waves, dawn-dusk asymmetry of occurrence, radial IMF.

\section{Introduction}

The low-frequency pulsations of the Earth's magnetic field, observed on the ground, have been attributed to hydromagnetic waves in the magnetosphere more than 40 years ago (Nagata et al., 1963; Kokubun and Nagata, 1965; Samson et al., 1971). Early observations of polarization variations in the Pc 5 frequency $(1.7-6.7 \mathrm{mHz}$ range) revealed the dependence of polarization on both the local time and the latitude of observations (Kokubun and Nagata, 1965; Samson et al., 1971; Samson and Rostoker, 1972). These observations led to the development of the coupling resonance theory (Tamao, 1965; Chen and Hasegawa, 1974; Southwood, 1974). Chen and Hasegawa (1974) and Southwood (1974) considered how fast surface waves, excited by the Kelvin-Helmholtz instability (KHI) on the magnetopause, could drive standing Alfvén waves on field lines of matching eigenfrequency (FLRs). Kivelson and Southwood $(1985,1986)$ presented the problem of explaining the occurrence of discrete frequency FLRs by proposing that radially standing fast cavity mode waves could be excited between the magnetopause and a turning point inside the magnetosphere. Since then, a large number of observational and theoretical works have delineated the features of ULF waves which are considered to be a mixture of transverse standing wave oscillations of the magnetic field lines and compressional oscillations of the magnetospheric cavity. However, unequivocal observations of magnetospheric cavity waves are actually rare (Hartinger et al., 2012).

Copyright (c) The Society of Geomagnetism and Earth, Planetary and Space Sciences (SGEPSS); The Seismological Society of Japan; The Volcanological Society of Japan; The Geodetic Society of Japan; The Japanese Society for Planetary Sciences; TERRAPUB.

doi: $10.5047 /$ eps.2012.12.013
Observational studies with the use of not only groundbased magnetometers, but also auroral HF radar, and satellites have focused on many features, including wave polarization, occurrence distributions, and the relationship with solar wind variations. Sets of multiple, discrete, magnetospheric ULF oscillations have been observed in HF radar (Ruohoniemi et al., 1991; Samson et al., 1991; Walker et al., 1992; Fenrich et al., 1995) and ground magnetometer measurements for more than a decade (Samson et al., 1992a; Ziesolleck and McDiarmid, 1994, 1995; Chisham and Orr, 1997; Villante et al., 2001). Because of the repeatability, global nature, and stability of the multiple discrete oscillations over several hours, the observations have been interpreted as manifestations of magnetospheric cavity and/or waveguide modes. In these models, the solar wind provides broadband energy that couples to the eigen modes of the magnetospheric cavity. Therefore the observed discrete frequencies depend on internal properties of the magnetosphere, principally the radial Alfvén speed profile (e.g., Samson et al., 1992a, b). Auroral latitude Pc 5 FLRs, supposedly driven by MHD waveguide/cavity modes, have been observed to occur often at discrete frequencies near 1.3, 1.9, 2.6, and $3.4 \mathrm{mHz}$ (e.g., Ruohoniemi et al., 1991; Samson et al., 1991; Walker et al., 1992; Ziesolleck and McDiarmid, 1994; Fenrich et al., 1995).

In a follow-up study, Ziesolleck and McDiarmid (1995) examined the occurrence rate of discrete Pc 5 frequencies observed by auroral latitude magnetometers at all local times and found a broad distribution across their analyzed frequency range $(1-5 \mathrm{mHz})$, with occurrence enhancements near $f=2$ and $3 \mathrm{mHz}$, and a smaller occurrence enhancement near $4 \mathrm{mHz}$. Chisham and Orr (1997) calculated the occurrence rate of discrete Pc 5 frequencies 
observed by mid-latitude magnetometers and found occurrence enhancements near $f=2.1,3.1,3.9$ and possibly 4.9 $\mathrm{mHz}$, largely in agreement with the observations of Ziesolleck and McDiarmid (1995). In contrast with the other statistical analyses, Baker et al. (2003) concluded that there were no enhancements in the occurrence distribution of discrete ground based pulsation frequencies in the Pc 5 frequency band that persisted across all 10 years (1989-1999). However, Viall et al. (2009) found that certain frequencies, specifically $f=1.0,1.5,1.9,2.8,3.3$, and $4.4 \mathrm{mHz}$, occur more often than do other frequencies over 10 years (19952005), in their analysis of observations at synchronous orbit.

Several observational studies (e.g., Rostoker and Sullivan, 1987; Ziesolleck and McDiarmid, 1995; Chisham and Orr, 1997) have demonstrated the dominance of forenoon pulsations over their afternoon counterparts, with classic FLR signatures much more prevalent in the morning sector. Moreover, Rostoker and Sullivan (1987) reported that a large fraction of the afternoon pulsations they observed were perhaps driven by impulses in the solar wind rather than the magnetopause instabilities thought to energize the morning sector pulsations. In a survey of Pc 5 pulsation events observed with the IMAGE array, Mathie et al. (1999) presented evidence of clear resonance signatures in the morning sector, but showed that FLR signatures were ill-defined closer to local noon, consistent with the scenario described by Mann et al. (1999).

Recent event studies have revealed the existence of highly periodic number density (and dynamic pressure) variations upstream in the solar wind that were followed by magnetospheric oscillations (Kepko et al., 2002; Kepko and Spence, 2003). These studies questioned the conclusion that observations of multiple, discrete ULF oscillations are necessarily manifestations of magnetospheric cavity modes. Since compressional variations observed at synchronous orbit in the daytime sector have only been used as proxy of magnetospheric response in these studies, more extensive observations are needed for conclusion. Another possible explanation for the occurrence of discrete ULF oscillations in terms of standing magnetospheric surface waves (or Krusukal-Schwarzschild-modes) has recently been suggested (Plaschke et al., 2009a).

FLRs are dominated in space by magnetic perturbations in the azimuthal direction (e.g., Kokubun et al., 1989). The magnetic field component of the fundamental mode waves has a node at the equator, which means that it is often difficult to detect the FLR waves only by the magnetic field measurement near the equator (Singer and Kivelson, 1979; Anderson et al., 1990). A few studies have been conducted on properties of plasma flow oscillations in the Pc 5 frequency range in the magnetosphere (Kokubun et al., 1977, 2000; Mitchell et al., 1990; Nakamura et al., 1994), while the FLR has been clarified by magnetic field observations (e.g. Kokubun et al., 1976; Singer et al., 1982; Kokubun, 1985). Use of plasma data is advantageous to detect FLR waves in the equatorial magnetosphere. In this study the analysis will be made for both low energy plasma and magnetic field data obtained by the Geotail spacecraft (GTL) in the equatorial region of the outer magnetosphere beyond
9 Earth's radii ( $R e)$. A single-spacecraft observation cannot achieve the spatial variations of the wave properties that multipoint ground observations can unravel. Therefore, we will also use ground observations of geomagnetic variations in event analysis.

\section{Data and Analysis}

The survey of Pc 5 waves in the outer magnetosphere was made by using GTL data obtained for six year period from December, 1994 to the end of 2000 when GTL was shifted to the near-Earth orbit with the apogee of $9 \mathrm{Re}$ and the perigee of $30 \mathrm{Re}$. In this study we mainly use the magnetic field and plasma data (Kokubun et al., 1994; Mukai et al., 1994) and supplementary the electric field measurement (Tsuruda et al., 1994). The analysis is made for 12 second time resolution data, since the average values for 4 spin periods (12.2 seconds) are available for the plasma data, ion density, ion temperature and flow velocity measurements. In visual inspection of data plots for magnetic field and plasma parameters regular ion flow oscillations in the Pc 5 frequency range are found to be observed in more than half of orbits near the dawn side magnetosphere. Ion flow oscillations are less frequent in the dusk sector as compared with those in the morning sector. The selection of wave events was based on plasma flow variations with amplitudes of larger than about $30 \mathrm{~km} / \mathrm{s}$ as a criterion.

In the following analysis the average field-aligned coordinates are used, since the direction of the ambient magnetic field changes along the orbit. The average magnetic field is defined in terms of sliding averages of 125 points (approximately 1525 seconds.) of observed magnetic field variations. In the average field-aligned coordinates, $\mathbf{e}_{z z}$ is parallel to the average field direction, $\mathbf{e}_{x x}$ is perpendicular to the magnetic field in the average magnetic meridian and $\mathbf{e}_{y y}=\mathbf{e}_{z z} \times \mathbf{e}_{x x}$. The direction of $\mathbf{e}_{x x}$ is nearly outward from the Earth, except for intervals of the tail-like field configuration. The complex FFT method is used to examine the spectrum and polarization of plasma flows and magnetic variations in the field-aligned coordinates. The FFT analysis is basically made for a 52 minutes interval (256 points data) which are selected from visual inspection of plasma flow and magnetic variations. Frequency and polarization of oscillations are mainly determined from power spectral densities (PSDs) of transverse velocity components $\left(V_{x x}\right.$ and $\left.V_{y y}\right)$, since evident peaks are not seen in the spectrum of magnetic variations in some cases. The labels, $V_{x x}$ $V_{y y}$, and $B_{x x}-B_{y y}$, shown in the figures in the following section indicate total PSDs of flow velocity and magnetic variations. The total PSD is the sum of left-handed (L) and right-handed (R) components of FFT spectra.

To examine the energy flow associated with Pc 5 waves the Poynting flux of perturbations in the average fieldaligned coordinates is calculated, assuming that the electric field perturbation is given by $\delta \mathbf{E}=-\mathbf{V} \times \mathbf{B}_{\mathrm{av}}$, where $V=\left(V_{x x}, V_{y y}, V_{z z}\right)$ and $\mathbf{B}_{\mathrm{av}}=\left(0,0, B_{\mathrm{av}}\right)$, neglecting the second order perturbation. The sliding averages of 61 points (12.5 min interval) of calculated Poynting fluxes are used to examine changes in energy flow associated with Pc 5 signals.

In event analyses we use multipoint ground magnetic ob- 
Table 1. List of geomagnetic station.

\begin{tabular}{|c|c|c|c|}
\hline \multirow[t]{2}{*}{ Station name } & \multicolumn{2}{|c|}{ Geomagnetic } & \multirow{2}{*}{$\begin{array}{c}\text { Magnetic local noon } \\
\text { UT }\end{array}$} \\
\hline & Latitude & Longitude & \\
\hline Davis (DVS) & -76.5 & 128.2 & 8.2 \\
\hline Hornsund (HRN) & 73.9 & 126.0 & 8.4 \\
\hline Bjornaya (BJN) & 71.4 & 123.3 & 8.6 \\
\hline Sodankyla (SOD) & 63.9 & 119.8 & 8.9 \\
\hline Tromso (TRO) & 67.2 & 116.3 & 9.0 \\
\hline Abisko (ABK) & 66.1 & 114.7 & 9.1 \\
\hline Mawson (MAW) & -73.2 & 110.1 & 9.4 \\
\hline Tjornes (TJO) & 66.5 & 72.3 & 11.7 \\
\hline Syowa (SYO) & -66.2 & 71.6 & 12.0 \\
\hline Leirvogur (LRV) & 65.2 & 67.7 & 12.0 \\
\hline Narsarsuaq (NAQ) & 69.9 & 38.6 & 14.2 \\
\hline Godhavn (GDH) & 78.6 & 33.1 & 14.5 \\
\hline Clyde River (CRV)* & 78.7 & 17.6 & 15.9 \\
\hline Iqaluit (IQA) & 72.3 & 14.6 & 16.2 \\
\hline Cape Dorset (CDR) & 73.5 & 1.7 & 16.9 \\
\hline Poste-De-La-Baleine (PBQ) & 66.8 & 358.3 & 16.9 \\
\hline Igloglik (IGL)* & 76.5 & 352.9 & 17.4 \\
\hline Coral Harbour $(\mathrm{CHB})^{*}$ & 73.7 & 350.5 & 17.6 \\
\hline Fort Churchill (FCC) & 68.3 & 333.3 & 18.5 \\
\hline Baker Lake (BLC) & 73.5 & 328.6 & 18.8 \\
\hline Gjoa Haven (GJO)* & 77.5 & 326.6 & 18.9 \\
\hline Cambridge Bay (CBB) & 76.9 & 310.6 & 19.8 \\
\hline Yellow Knife (YKC) & 68.9 & 299.3 & 20.8 \\
\hline College (CMO) & 64.6 & 256.5 & 23.7 \\
\hline Huancayo (HUA) & -1.99 & 357.0 & 16.8 \\
\hline Bangui (BNG) & 4.1 & 91.5 & 10.7 \\
\hline M'Bour (MBO) & 19.8 & 57.8 & 12.2 \\
\hline Memambetsu (MMB) & 35.4 & 211.8 & 14.6 \\
\hline Fredericksburg (FRD) & 48.0 & 353.9 & 17.3 \\
\hline Ottawa (OTT) & 55.2 & 355.9 & 17.1 \\
\hline
\end{tabular}

servations to examine the ground conjugacy and longitudinal scale size of waves observed in the magnetosphere. The data (1 minute resolution) from standard geomagnetic stations are mainly examined in this analysis. Spectra of geomagnetic variations are calculated by using the deviations from the 31 point sliding average of one minute data in the horizontal plane ( $X$ and $Y$ components). The total PSD in the $X-Y$ plane is estimated for ground data as in the case of GTL data. Total PSDs are used in comparison of spectral characteristics between the ground and magnetosphere. The high-resolution data from the MACC array (Engebretson et al., 1995) are also utilized to analyze spectral structures of magnetic fluctuations at high latitudes for specific intervals. The list of major stations near the cusp and auroral latitudes is given in Table 1. We also refer to data from Huancayo (HUA), Bangui (BNG) and M'Bour (MBO) in the equatorial region and other stations in low latitudes.

The PC index (Kokubun, 1972) is used to check the magnetic activity in the polar cap region as well as AE index available from the WDC for Geomagnetism, Kyoto University. The PC index is defined as the magnitude of deviations in the horizontal component from the quiet day level at stations in the polar cap and is used as a proxy of the two cell convection pattern (Kamide and Kokubun, 1996). In addition to geomagnetic stations listed in Table 1, data from four stations, Alert (ALE), Resolute Bay (RES), Thule (THL) and Dumont d'Urville (DRV) in the central polar cap are used to calculate PC indices. ACE and Wind spacecraft data as well as the OMNI (Combined 1AU IP Data; Magnetic and Solar Indices) data, which are available from CDAWeb, NASA, are used to examine the solar wind and interplanetary magnetic field (IMF) conditions. ACE data are utilized in event analysis.

Figure 1 represents an example of the GTL data obtained in the morning sector $\left(-6 R_{E}<X_{\mathrm{GSM}}<6 R_{E}\right)$ on May 19 , 1999. Two temperatures $\left(T_{y}\right.$ and $\left.T_{z}\right)$ are plotted together in $T_{p}$ panel, where $T_{y}$ is the temperature perpendicular to the spin axis and $T_{z}$ is the temperature along the spin axis. Since the spin axis of GTL is inclined about 10 degrees to the magnetic field, $T_{y}$ roughly indicates the temperature perpendicular the ambient magnetic field.

A train of wave packets of amplitude larger than $30 \mathrm{~km} / \mathrm{s}$ in the Pc 5 frequency range is clearly seen in an interval of $10-15 \mathrm{~h}$. The frequency of the major spectral peak is confirmed to be almost constant, $3.5 \mathrm{mHz}$, in this five hour interval, though the spacecraft moved along the L-shell of 


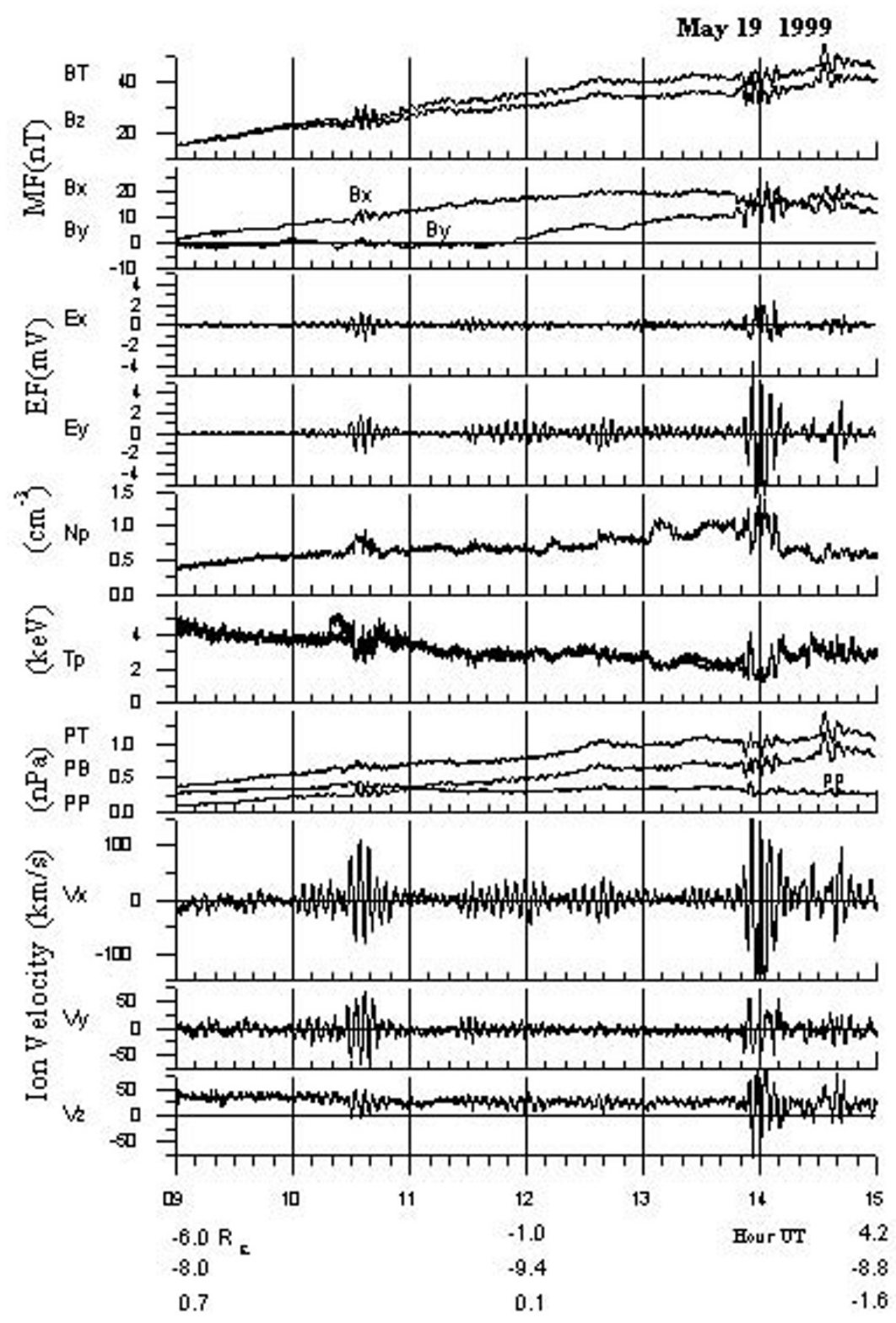

Fig. 1. An example of Geotail data plot. Magnetic field and ion velocity components are plotted in GSM coordinates together with electric field variations in spacecraft coordinates. The ion density $\left(N_{p}\right)$, ion temperatures $\left(T_{p}\right)$, magnetic pressure $\mathrm{PB}\left(\left(B_{T}\right)^{2} / 2 \mu_{0}\right)$, plasma pressure $\mathrm{PP}\left(N_{p} k T\right)$ and the total pressure PT $(\mathrm{PP}+\mathrm{PB})$ are shown in the middle part of the figure.

$\sim 10.5$ by $40^{\circ}$ in longitude. Spectra and ground correlations will be discussed in more detail in the next section. In Fig. 2(a) we note some features of the transverse waves. The absolute value of the normalized amplitude, $d B_{T} / B_{\mathrm{av}}$, was often less than 0.1 . It seems to be noteworthy that the envelope curve of flow variation $\left(V_{x}\right)$ is similar to that of PC. This suggests that the development of Pc 5 oscillations has a close correlation with the magnetospheric convection.

Fluctuations in the dynamic pressure of solar wind are shown in the top forth panel of Fig. 2(a). Kepko and Spence (2003) argue that solar wind density fluctuations often alter slowly the size of the magnetospheric cavity leading to the appearance of multiple, discrete magnetospheric oscillations. However, observed dynamic pressure fluctuations are not so large and periodic as to account for magnetospheric oscillations in this case. On the other hand, discon- tinuous changes of IMF seems to be correlated with changes in wave property and ground magnetic variations. In order to examine oscillating features of IMF, the power spectral density of IMF variations is calculated by the same procedure as the analysis of magnetospheric oscillations. The field-aligned coordinates are defined based on the sliding average of 125 point data of 16 second resolution (ACE data), and the rotational spectrum in the plane perpendicular to the average magnetic field is estimated The result of IMF analysis will be given in the next section.

Figure 2(b) represents an example of time variations of Poynting flux, and energy flow vectors perpendicular to the ambient magnetic field. The average of the field-aligned flux $\left(P_{z z}\right)$ is small except for a wave packet around $14 \mathrm{~h}$, indicating the feature of standing wave. On the other hand, fluxes perpendicular to the ambient magnetic field are larger 


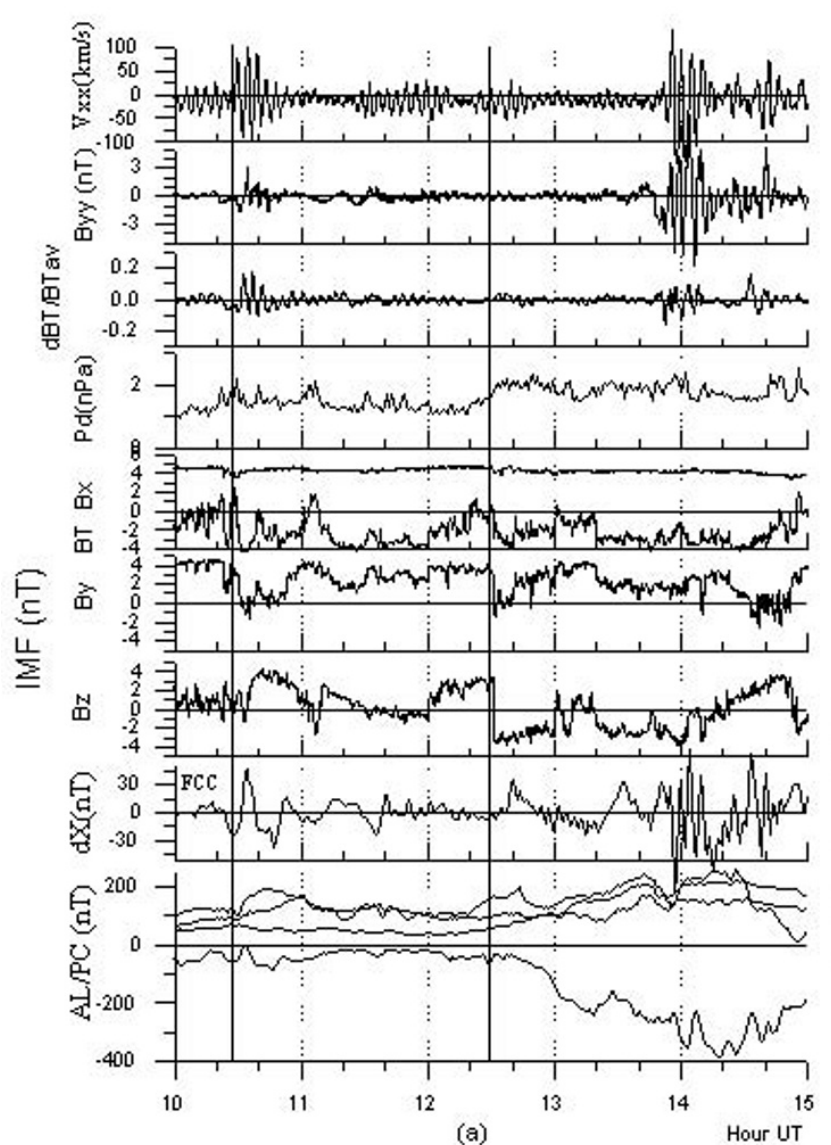

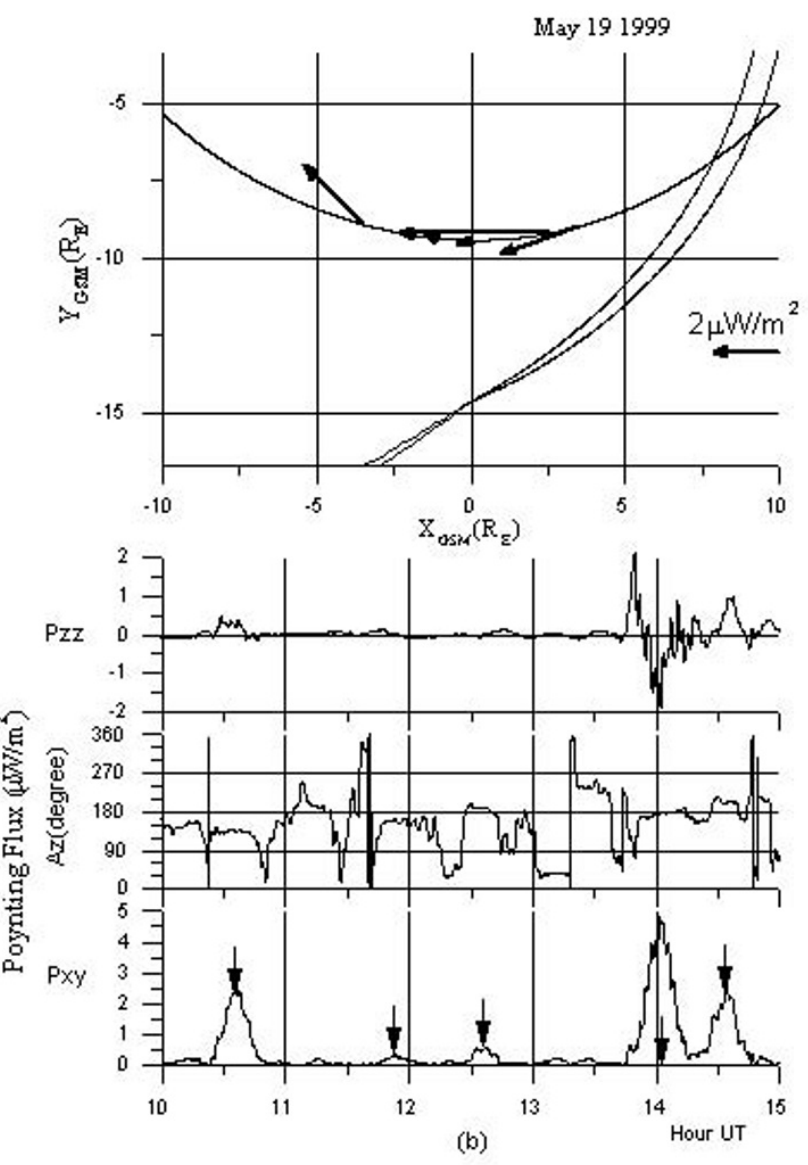

Fig. 2. (a) GTL data $\left(V_{x x}, B_{y y}\right.$ and normalized amplitude, $\left.d B_{T} / B_{\text {av }}\right)$ in field-aligned coordinates, solar wind dynamic pressure $\left(P_{d}\right)$ and IMF variations (ACE data, time shifted by $66.3 \mathrm{~min}$ ) are shown along with high-pass-filtered magnetic variations at FCC. The bottom panel shows AL and PC indices (ALE, THL and CBB). (b) An example of time variations of Poynting flux, and energy flow vectors perpendicular to the ambient magnetic field. $P_{z z}$ denotes the field-aligned flux and $P_{x y}$ is the magnitude of energy flow vector in the $x x-y y$ plane. The top panel shows energy flow vectors at times of peaks of five wave packets indicated by arrows.

than the field-aligned flux. The direction of the Poynting vector in the plane perpendicular to the ambient field is anti-sunward with a small earthward component. Accordingly, the energy of oscillation is transferred tailward by the coupling with the compressional component. This result is consistent with the analysis by using the electric field measurement by GTL (Sakurai et al., 2001). The statistics of Poynting flux will be given later.

\section{Transverse and Compressional Waves}

The features of transverse waves are mainly examined in this paper. The amplitude ratio of compressional variations to the average background field, normalized amplitude $\left(d B_{T} / B_{\text {av }}\right)$, and the anti-phase relation between the ion and magnetic pressures were taken into account to distinguish transverse waves from compressional waves. The following diamagnetic relation holds for wave perturbations with a high azimuthal wave number, $m$ (Southwood, 1976).

$$
\Delta b_{\|}+\mu_{0} \delta p / B_{T}=0 .
$$

In other words, perturbations of the ion pressure and magnetic pressures should be anti-correlated for large mnumber waves. Since the transverse wave of FLR-type has a low wave number (e.g., Chisham and Orr, 1997), the dia- magnetic relation would not be applied in the case of FLRs. To check the diamagnetic relation field magnitude deviations were compared with values estimated from plasma measurements.

$$
\delta b=-\mu_{0} \delta p / B_{\text {av }}
$$

here, $\delta p$ is estimated as the deviation from the 125 point sliding average of observed plasma pressure.

Figure 3 shows GTL data observed on February 9-10, 1999 , when the spacecraft was on a skimming orbit nearly along the daytime magnetopause. Oscillations of plasma flow with amplitudes of several tens $\mathrm{km} / \mathrm{s}$ were observed in the 6-9 h local time sector on February 9. Magnetic activity was low during this interval $\left(K_{\mathrm{p}}\right.$ index: $\left.0-0+\right)$. The GTL spacecraft was in the magnetosphere, except for a very short interval (25 seconds) around 12:31, as indicated by impulsive changes in $B_{T}$ and $V_{x}$ in Fig. 3(a). These variations was associated with a transient magnetosheath entry.

The characteristic difference of wave properties between the dawn and dusk sides are evident in the both magnetic and ion flow variations in the upper four panels. Regular flow oscillations were observed on the morning side from

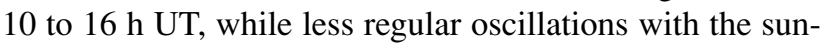
ward component of average flow were detected on the dusk 

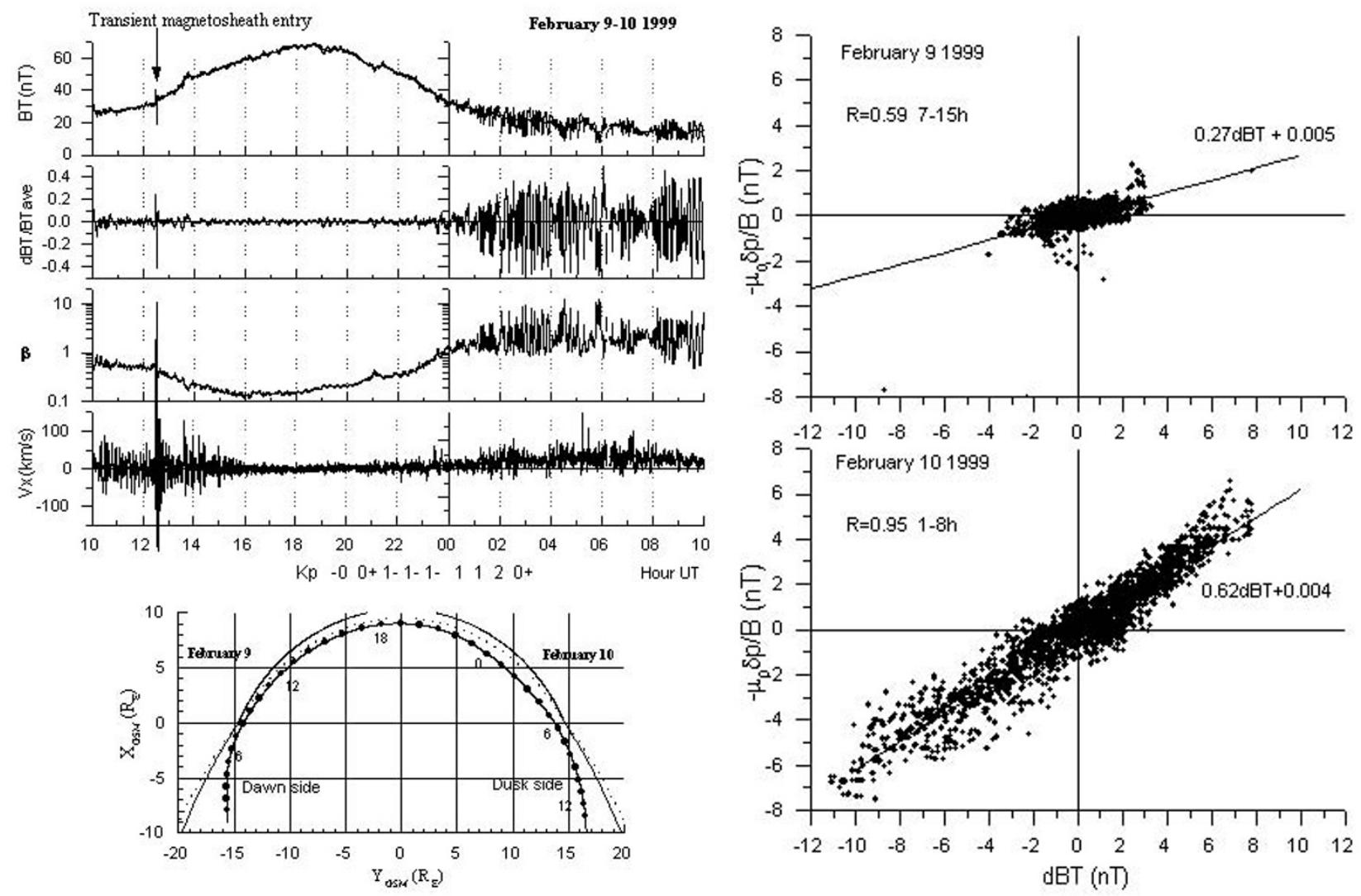

(a)

(b)

Fig. 3. (a) GTL data obtained on a skimming orbit nearly along the magnetopause on February 9-10, 1999. GTL was always in the magnetosphere except for a short time interval at 12:31. Fairly regular oscillations of plasma flow with amplitudes more than $50 \mathrm{~km} / \mathrm{s}$ were observed in the morning sector. In contrast, wave forms were less regular in the afternoon sector. A remarkable contrast between the morning and afternoon sides is notable in normalized amplitude and plasma $\beta$. (b) Scattered plots of quantities estimated from the diamagnetic relation versus observed variations of the field magnitude, $d B_{T}$.

side from 0 to $13 \mathrm{~h}$ on February 10. It is affirmed by plasma measurements that GTL did not entry into the LLBL plasma on the afternoon side, since the plasma temperature and number density were in ranges of $2 \mathrm{kev}<T_{p}<8 \mathrm{kev}$, and $N_{p}<0.8 / \mathrm{cm}^{3}$, respectively. The normalized amplitudes of field magnitude variation are plotted in the second panel of Fig. 3(a). Amplitudes of the field magnitude fluctuation in the afternoon sector were fairy large to the background field, while normalized amplitudes were small in the morning sector. It is found that magnitude variations associated with large flow oscillations are smaller than 0.1 before noon. On the other hand, large fluctuations of normalized amplitude more than 0.4 are apparent in the afternoon-dusk region. The interval after $0 \mathrm{~h}$ UT is also characterized by plasma $\beta\left(N_{p} k T /\left(\left(B_{T}\right)^{2} / 2 \mu_{0}\right)\right)>1$. The regular oscillation with a small compressional component, observed in the morning sector, is the typical feature of the FLR in the outer magnetosphere. Compressional oscillations observed on the afternoon side on February 10 could be categorized as those studied in previous works (e.g., Zhu and Kivelson, 1991; Constantinescu et al., 2009).

In Fig. 3(b) quantities estimated from the diamagnetic relation, $-\mu_{0} \delta p / B_{\text {av }}$ are plotted versus observed $d B_{T}$ for magnetic variations in the morning and afternoon regions, respectively. The upper figure shows a scatter plot for $7-$
$15 \mathrm{~h} \mathrm{UT}$ on February 9 when regular flow oscillations in the Pc 5 frequency range were observed. The lower panel shows a scatter plot for 1-8 h UT on February 10. Calculated values have a high correlation with observed fluctuations in the case of compressional signals in the afternoon sector. The correlation coefficient is 0.95 and a slope of regression line is 0.62 . Approximately $60 \%$ of observed field fluctuations are attributable to the density variations, as evident from the regression analysis. On the other hand, the correlation coefficient is 0.59 , and the slope of regression line is 0.27 , in case of February 9. These values are smaller as compared with those of February 10 case.

As mentioned above, transverse waves of the FLR type examined in this analysis were selected by the inspection of plasma flow oscillations. We also checked the normalized amplitudes of field magnitude. Normalized amplitudes of selected waves are mostly less than 0.1 .

\section{Event Analysis}

In the previous section the selection criteria and the Poynting flux of the transverse wave were briefly discussed, as examples of events observed on February 9 and May 19, 1999. The May 19 event appears to show that the occurrence of substorm causes changes in wave property. To illustrate further substorm effects on wave property two large 


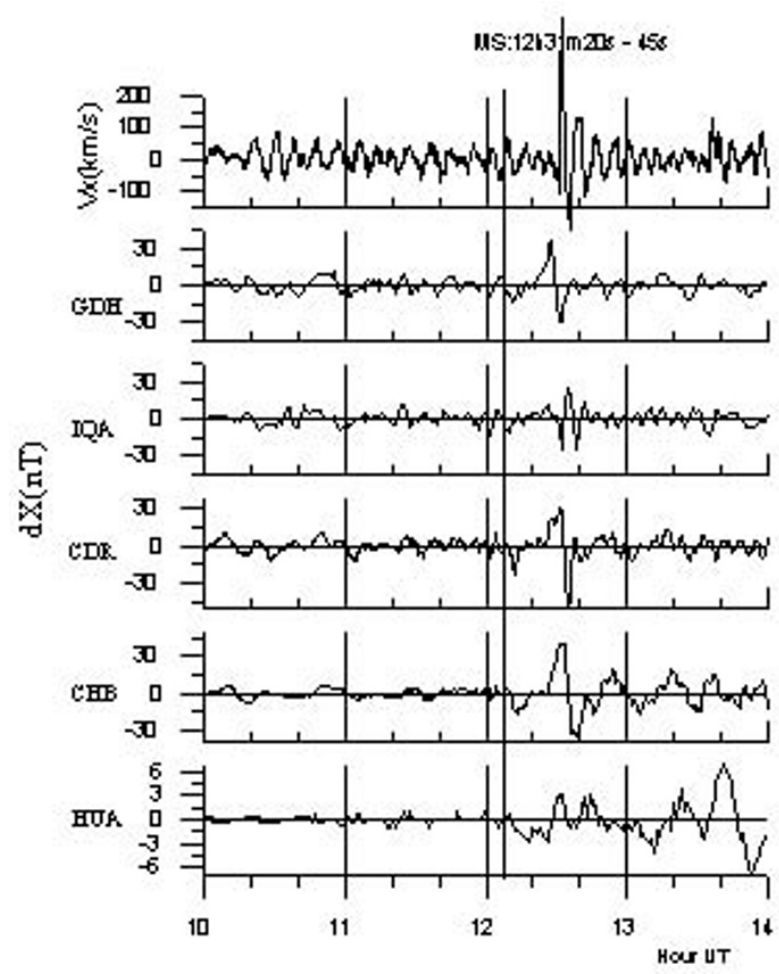

(a)

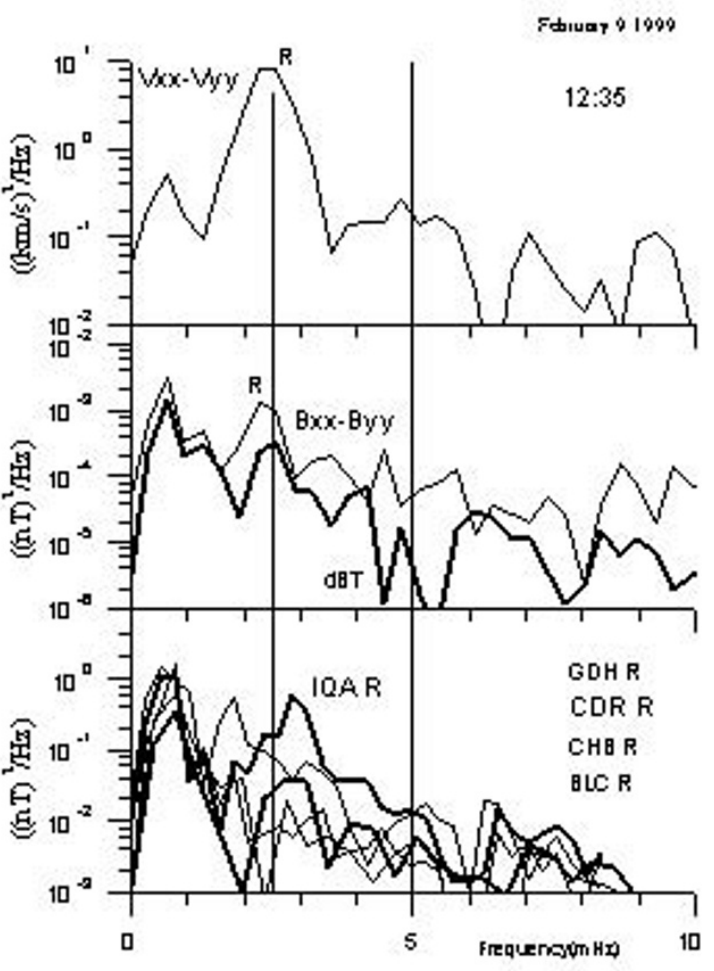

(b)

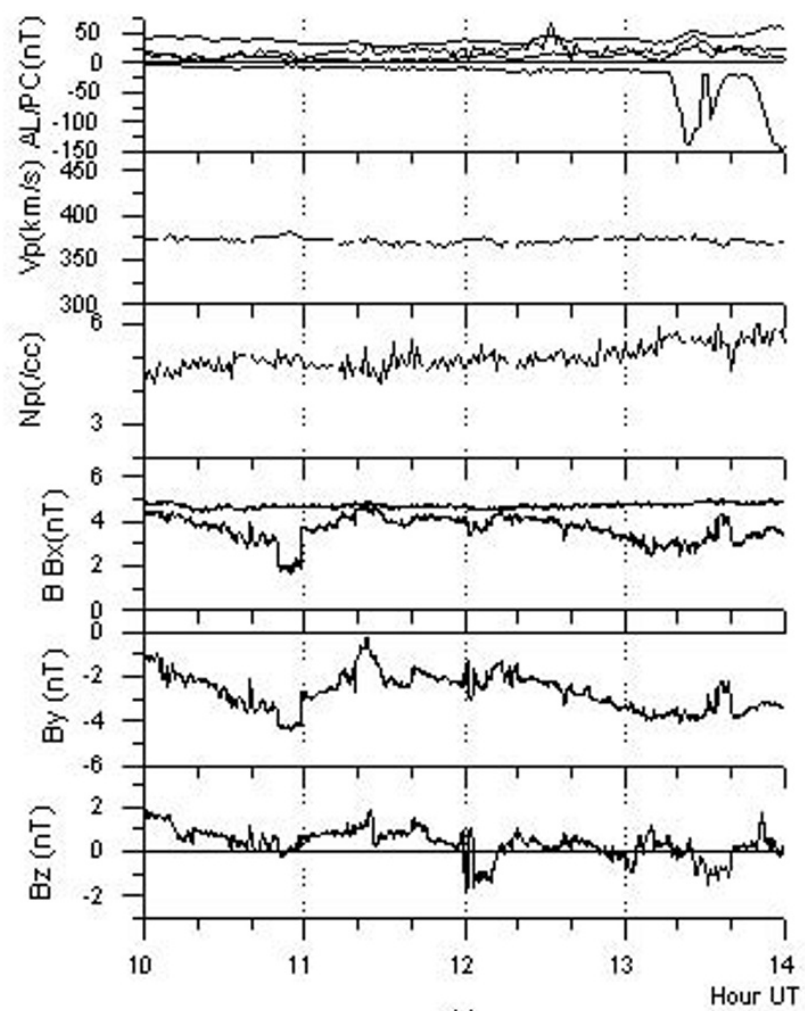

(c)

Fig. 4. (a) High-pass filtered geomagnetic variations at high latitude stations GDH, IQA, CDR, CHB and at HUA in the dip equator during 10-14 $\mathrm{h}$ on February 9. (b) Power spectral densities (PSDs) on the ground and plasma flows and magnetic variations observed by GTL after the transient stay in the magnetosheath. Total PSDs at five stations, IQA, GDH, CDR, CHB and BLC, in cusp latitudes are plotted in the bottom panel. The label R, indicated with station codes, represents the polarization mode at major spectral peaks. (c) Plots of solar wind and IMF observed by the ACE spacecraft (time shifted by $72 \mathrm{~min}$ ) along with AL/PC indices. Three lines show PC indices derived from geomagnetic variations at IQA, ALE and DRV. 
amplitude events are examined in relation to solar wind and IMF conditions. A series of wave trains for several hours were observed in the morning sector in the previous two examples. On the other hand, we noted that wave trains of long duration, such as seen in these examples, seldom occurred in the afternoon-dusk sector. Plasma flow oscillations observed on the dusk side of the outer magnetosphere are isolated of which duration is approximately one hour. Two wave events observed in the dusk sector are analyzed to exemplify the dawn-dusk asymmetry of wave characteristics. The longitudinal scale and ground conjugacy of waves detected on GTL are also discussed in comparison with ground magnetic data in the following analysis.

\subsection{February 9, 1999}

The transient entry, seen in Fig. 3, appears to give us information for the resonance region of field line oscillation, since waves were observed before and after the magnetosheath entry of GTL. Transverse waves with the frequency of $1.9 \mathrm{mHz}$ and amplitudes of $\sim 50 \mathrm{~km} / \mathrm{s}$ continued for about two hours from 10:20 before the magnetosheath entry. The polarization of waves was of left-handed (L) mode during this interval. On the ground weak pulsations were observed at HRN, GDH and CDR in cusp latitudes.

Figure 4(a) represents high-pass filtered records of geomagnetic variations. FFT spectra of geomagnetic variations and plasma flows after the sheath entry are shown in Fig. 4(b). Magnetic field variations in cusp latitudes began around 12:09 preceding magnetic impulses related to the sheath entry. The spectra of plasma flow and magnetic field in 52 minutes interval from 12:35 showed the right-handed (R) polarization at peak frequency of $2.6 \mathrm{mHz}$, while the polarization of waves in later time from $\sim 13: 30$ was lefthanded. This suggests that the polarization reversal from the $\mathrm{R}$ mode to $\mathrm{L}$ mode occurred at some distance from the magnetopause. Namely, the field line resonance was excited in the region close to the magnetopause, though spectra observed at ground stations do not necessarily showed a clear peak. The spectral peak at CDR and IQA situated in cusp latitudes roughly agrees with the $2.6 \mathrm{mHz}$ peak in GTL spectra.

The solar wind and IMF data, and AL index shown in Fig. 4(c) represent that the geomagnetic activity and the solar wind were very quiet before the transient magnetopause entry. The solar wind speed and ion density were almost constant, $375 \mathrm{~km} / \mathrm{s}$ and $5 / \mathrm{cm}^{3}$. This would not be a favorable condition for the excitation of ULF waves and magnetopause deformation by KHI and/or direct driven process. Instead, a small but sharp southward turning of the IMF $B_{z}$ component around $12 \mathrm{~h}$ would be related to the sheath entry of GTL.

\subsection{May 19, 1999}

The major frequency of wave trains observed by GTL was almost stable in frequency range of $3.1-3.8 \mathrm{mHz}$ throughout five hours from $10 \mathrm{~h}$ UT on. Spectra which were calculated for three intervals from 10:10, 11:25, and 12:20 have the major peak at $3.5 \mathrm{mHz}$. Figure 5(a) and (b) show power spectra of plasma flow and magnetic field observed by GTL in the intervals from 13:50, and corresponding spectra at ground stations, and at GOES 8 on synchronous orbit. This interval corresponds to the maxi- mum phase of substorm (Fig. 2). Note that GTL spectral peaks do not always agree with each other among the transverse velocity and magnetic field, and the field magnitude. The polarization of oscillations at GTL is left-handed. The three peaks, $2.2,3.1$ and $3.8 \mathrm{mHz}$, of GTL spectra correspond to those of ground spectra shown in Fig. 5(b). The major spectral peak observed at synchronous orbit agrees with a subpeak of GTL spectra at $2.2 \mathrm{mHz}$, and spectral peaks at ground variations. The third panel from the top in Fig. 5(b) shows spectra observed in the afternoon sector on the ground, including the spectrum at HUA in the dip equator. L-mode waves were observed at auroral oval stations in both morning (FCC and PBQ) and afternoon sectors (NAQ, LRV and TRO), though the spectral density is smaller in the afternoon sector. The polarization at IQA, CDR, and BLC in magnetic latitudes of $\sim 73$ degree is of the $\mathrm{R}$ mode, indicating the polarization reversal occurred along cusp latitudes.

The spectrum of transverse IMF fluctuations, shown in the bottom panel of Fig. 5(b), has several peaks which correspond to those of GTL and ground variations. Good correlation with magnetospheric spectra is not observed in other three intervals. The azimuthal angle of IMF was more radial within $150-170^{\circ}$ in the period from $13: 50$, as compared with other three periods. Incidence of Alfvénic fluctuations to the magnetosphere would be favorable under radial IMF conditions. It is speculated that Alfvénic fluctuations contribute to transfer the solar wind energy to drive magnetospheric oscillations through the reconnection process. A similar correspondence between IMF and ground spectra are seen in other events discussed later.

\subsection{March 7, 1999}

Properties of transverse waves are further examined in relation to ground magnetic variations and the solar wind condition. The next example is one of the largest amplitude events which was observed in the local time sector of 6-8 hour on March 7, 1999 (Fig. 6). The temperature anisotropy $T_{A}$ plotted in Fig. 6 is defined as $T_{A}=2\left(T_{y}-T_{z}\right) /\left(T_{y}+T_{z}\right)$. A series of pulsation trains was observed during three hours from 13:35. Waveform and frequency were changing with time, different from the previous case. This change appears to be related to the development of substorm. Geomagnetic indices, $\mathrm{AL}$ and $\mathrm{PC}$, do not indicate significant activity in the time interval of first wave packet, and then show the growth phase feature from about 14:40. The temperature anisotropy was gradually increasing from 15:28 with the development of substorm, namely the perpendicular temperature became larger than the parallel temperature. The average sunward component $\left(V_{x}\right)$ of the plasma flow enhanced after the beginning of growth phase, and the wave frequency became higher. The plasma $\beta$ before the substorm onset was less than 1 .

Figure 7 represents periodic magnetic field variations at three stations, NAQ, FCC and YKC in the auroral oval, $\mathrm{CHB}$ in the polar cap boundary, and HUA in dip equator along with IMF data from ACE spacecraft (time shifted by 45.2 minutes). The foot point of GTL orbit (available from http://darts.isas.jaxa.jp/stp/geotail/) moved along a circle of approximately 73 degrees in geomagnetic latitude. Largest pulsation with amplitude of $\sim 100 \mathrm{nT}$ was observed at CHB, 


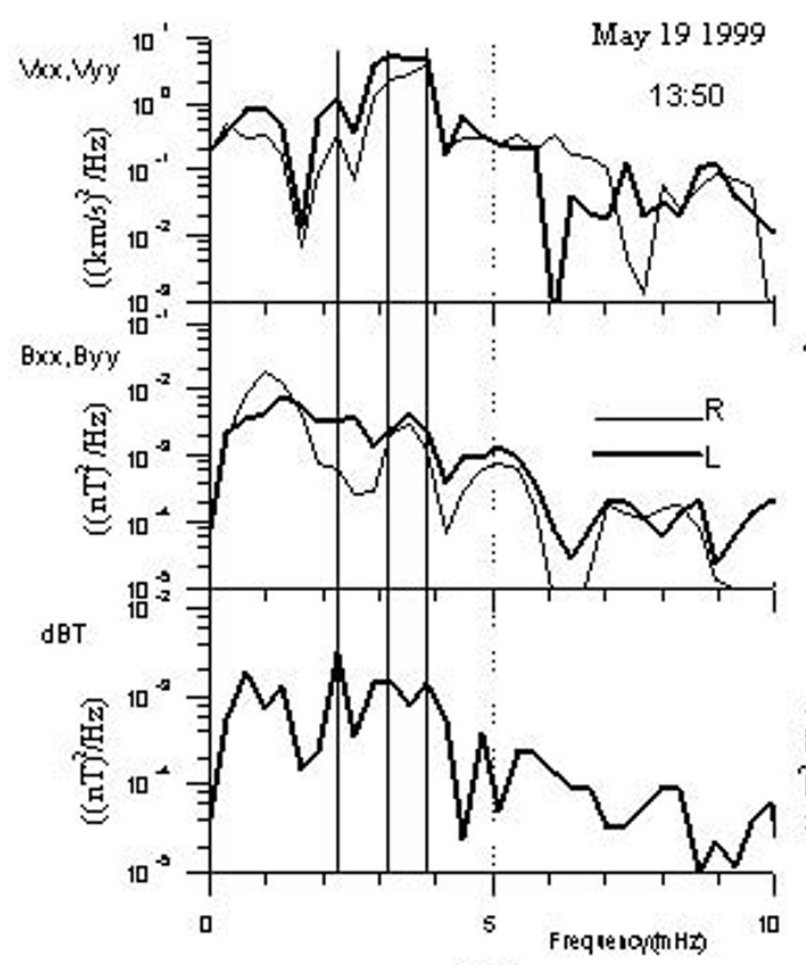

(a)

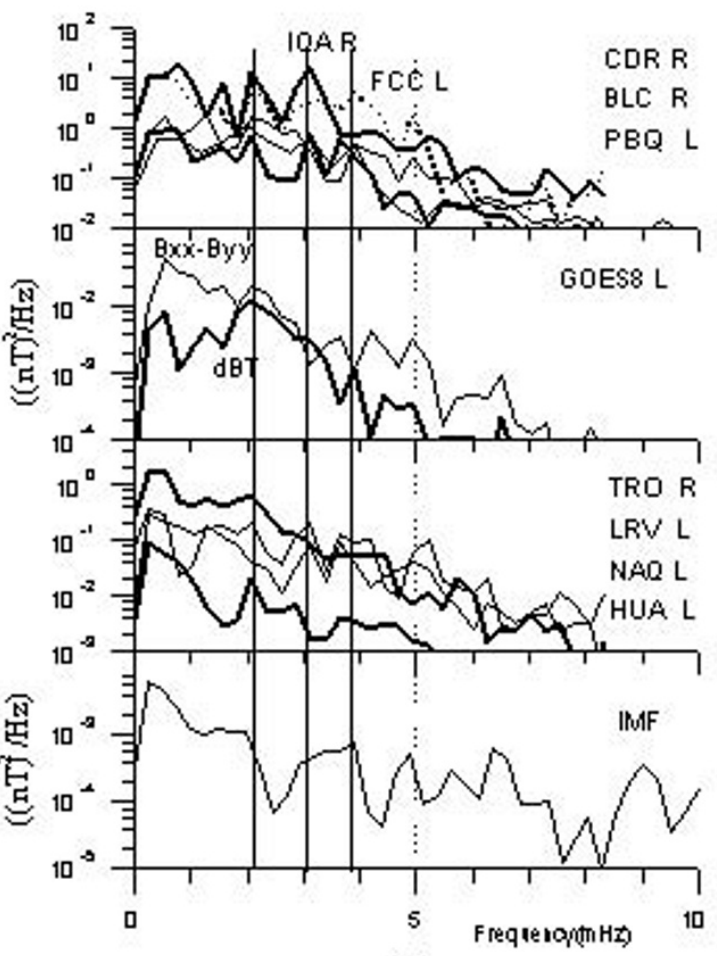

(b)

Fig. 5. (a) PSDs of plasma flow and magnetic field oscillations in the time interval of 52 minutes from 13:50 on May 19, 1999. Rotational PSDs are plotted separately for the right-handed (R) and left-handed (L) components of transverse flow and magnetic field. (b) Spectral estimates for ground and GOES 8 data. Ground spectra are plotted separately for stations in the morning and afternoon sectors (top and third panels). The bottom panel shows total PSD of IMF variations obtained from ACE data (time shifted by $66.3 \mathrm{~min}$ ). The labels, $\mathrm{L}$ and R, indicated with station codes represent polarization modes at major spectral peaks.

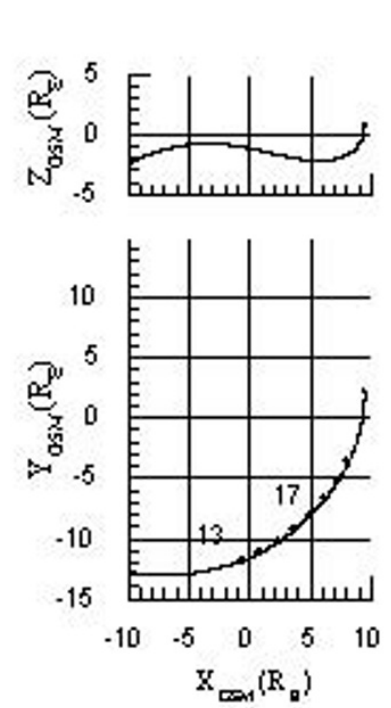

March 71999

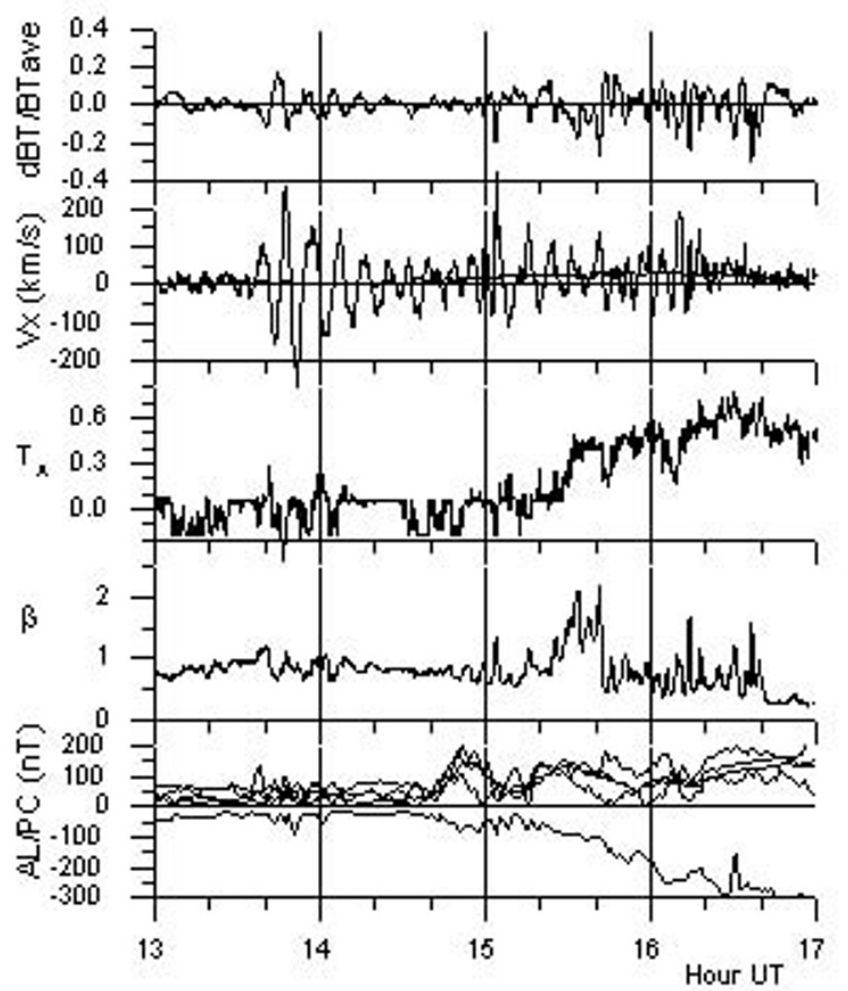

Fig. 6. GTL data and ground magnetic activity on March 7, 1999. AL and PC (ALE, BLC, IQA and THL) indices are plotted in the right bottom panel. The sliding averages of $V_{x}$ is plotted together with $V_{x}$. The GTL orbit is shown in the left. 


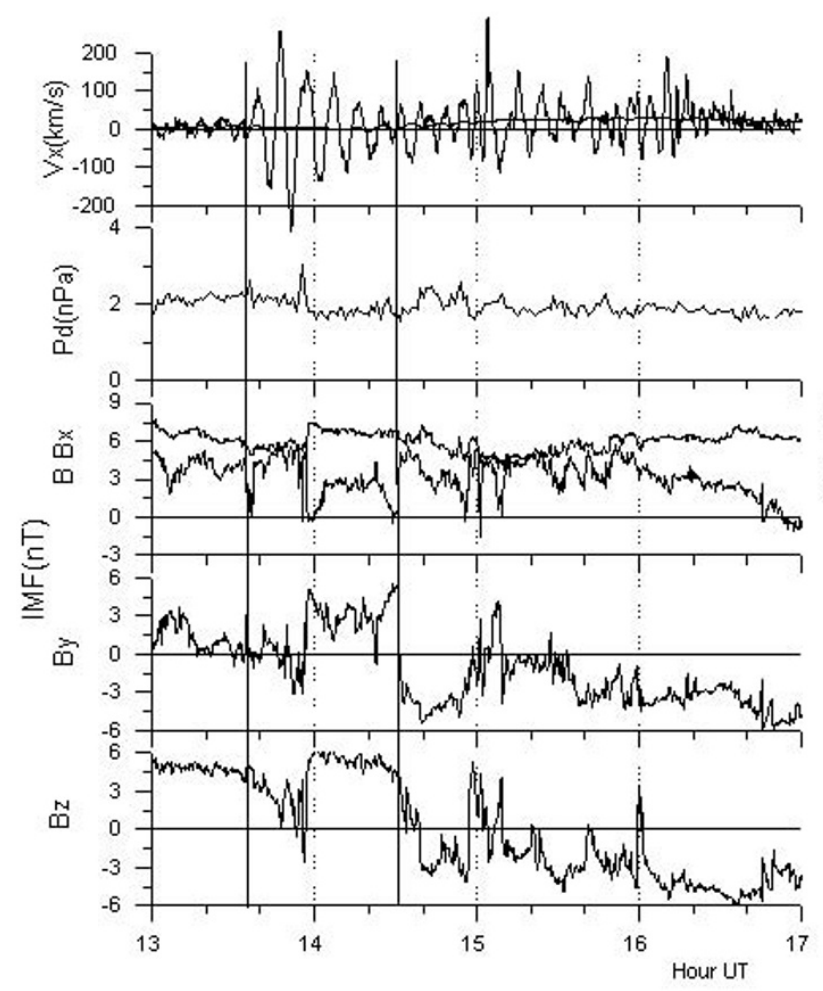

(a)

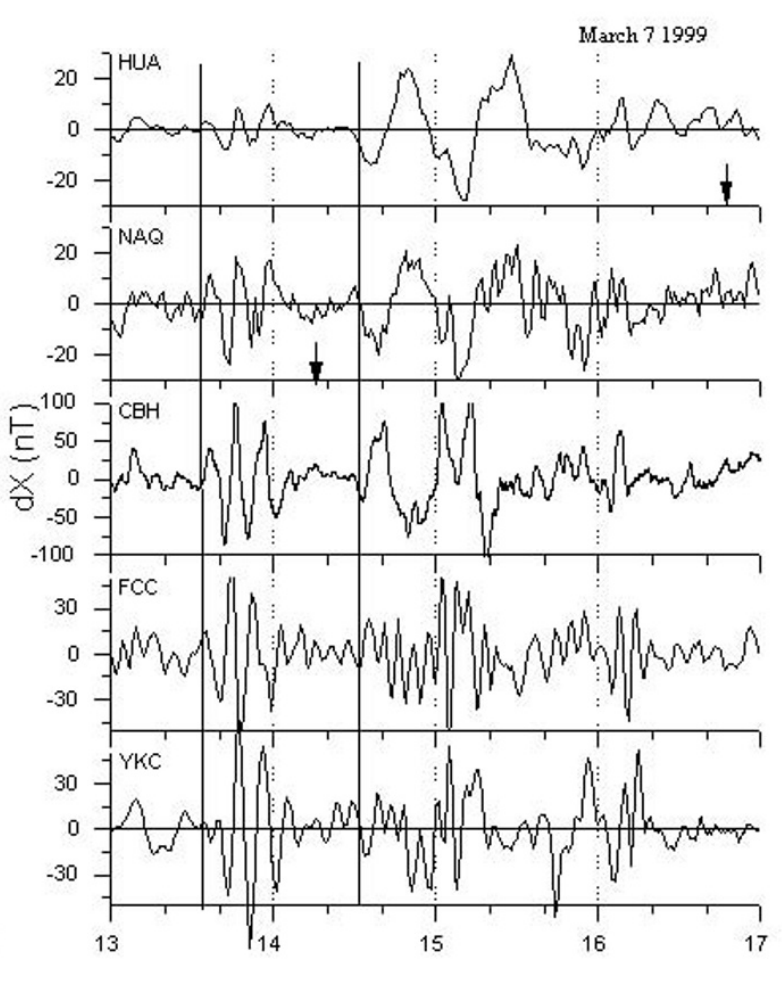

(b)

Fig. 7. (a) Waveforms of ion flow at GTL, solar wind dynamic pressure and IMF variations in GSM coordinates. (b) High-pass filtered records from five stations, HUA in dip equator, CHB in cusp latitudes, NAQ, FCC and YKC in the auroral oval. The arrows indicate local magnetic noon at HUA and NAQ. Note that the waveform at HUA is very similar to those at high latitude stations.

the closest station to the conjugate point of GTL, in the first interval. The speed and dynamic pressure of solar wind were almost constant, about $500 \mathrm{~km} / \mathrm{s}$ and $2 \mathrm{nPa}$ in the interval shown in the figure. Significant spectral components could not be found in the dynamic pressure of solar wind corresponding to pulsation frequencies. On the other hand, discontinuous IMF changes appears to be related to the onset of wave and changes in waveform both in space and on the ground. Therefore rotational spectra of IMF were calculated as in case of May 19 event.

Spectral analysis of GTL data for three intervals from $13: 35,14: 40$ and 15:45 indicates that the major spectral peak is at $1.6 \mathrm{mHZ}$ in the former two intervals, and shifted to $2.6 \mathrm{mHz}$ in the third interval. Secondary spectral peaks are also noted at $3.2 \mathrm{mHz}$ and $4.7 \mathrm{mHz}$ in the first interval, as shown in the right panel of Fig. 8(a). Spectra of ground magnetic variations at high latitudes for the first interval are presented in the right panel of Fig. 8(a). Ground spectra are separately plotted for three groups of stations in the polar cap, in cusp latitudes and in auroral latitudes. The common peak is found at $1.6 \mathrm{mHz}$ in ground spectra in a wide region of geomagnetic longitude from $310^{\circ}$ to $33^{\circ}$ (GDH through $\mathrm{CBB}$ ) in the forenoon to noon sector. The polarization is right-handed in the polar cap and cusp region, while lefthanded at auroral latitude stations. The polarization at the main peak of spectra at GTL is almost linear.

Note that spectra shown in the right bottom panel of Fig. 8(a) are those of stations situated along the auroral oval on the afternoon side of about 60 degrees in longitudinal width. Though PSDs are one-order of magnitude smaller than those in the morning region, the polarization at the spectral peak in the afternoon sector is left-handed, same as that in the morning region. This indicates that a global oscillation occurs simultaneously in both the morning and afternoon regions. We confirmed that pulsations with amplitudes of 2-3 nT and waveforms similar to those in high latitudes were also observed in middle latitudes. Their spectral characteristics are very similar to those in higher latitudes. The largest PSD in middle and low latitudes was observed at Huancayo in the dip equator, indicating that a global ionospheric current with the equatorial enhancement was induced in association with the wave excitation in the magnetosphere. The $1.6 \mathrm{mHz}$ peak of PSD at HAU is a factor 5 larger than that at $\mathrm{MBO}$ (geomagnetic latitude: $19.9^{\circ}$ ). As for secondary spectral peaks, $4.7 \mathrm{mHz}$ peak seem to be observed in cusp and polar cap regions. However, the 3.2 $\mathrm{mHz}$ peak is not evident in the ground spectra.

The major peak at GTL in the second interval from 14:40 is same as the $1.6 \mathrm{mHz}$ peak in the former interval, while secondary peaks appeared around $3.2 \mathrm{mHz}$ and $4.2 \mathrm{mHz}$ (Fig. 8(b)). Corresponding shifts also appear in the ground spectra. The polarization in the afternoon sector changed to the R-mode. In the following interval the major peak shifted to $2.6 \mathrm{mHz}$ with a secondary broad peak around 4.3 $\mathrm{mHz}$, while the major peak is at $4.3 \mathrm{mHz}$ in the compressional component. Corresponding spectra at $2.6 \mathrm{mHz}$ on the ground were observed only in the morning region. These sequential changes appear to be due to magnetospheric con- 

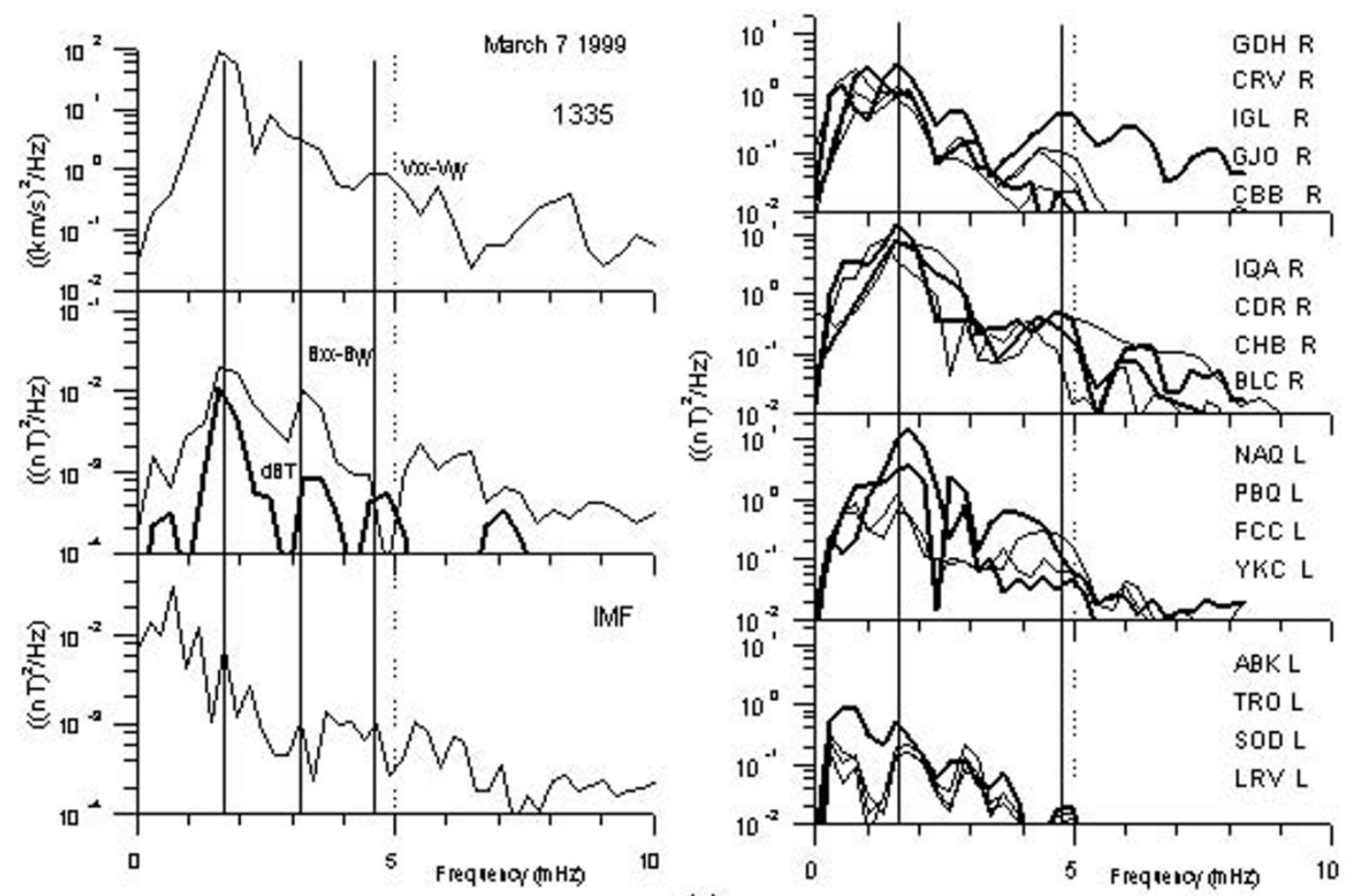

(a)
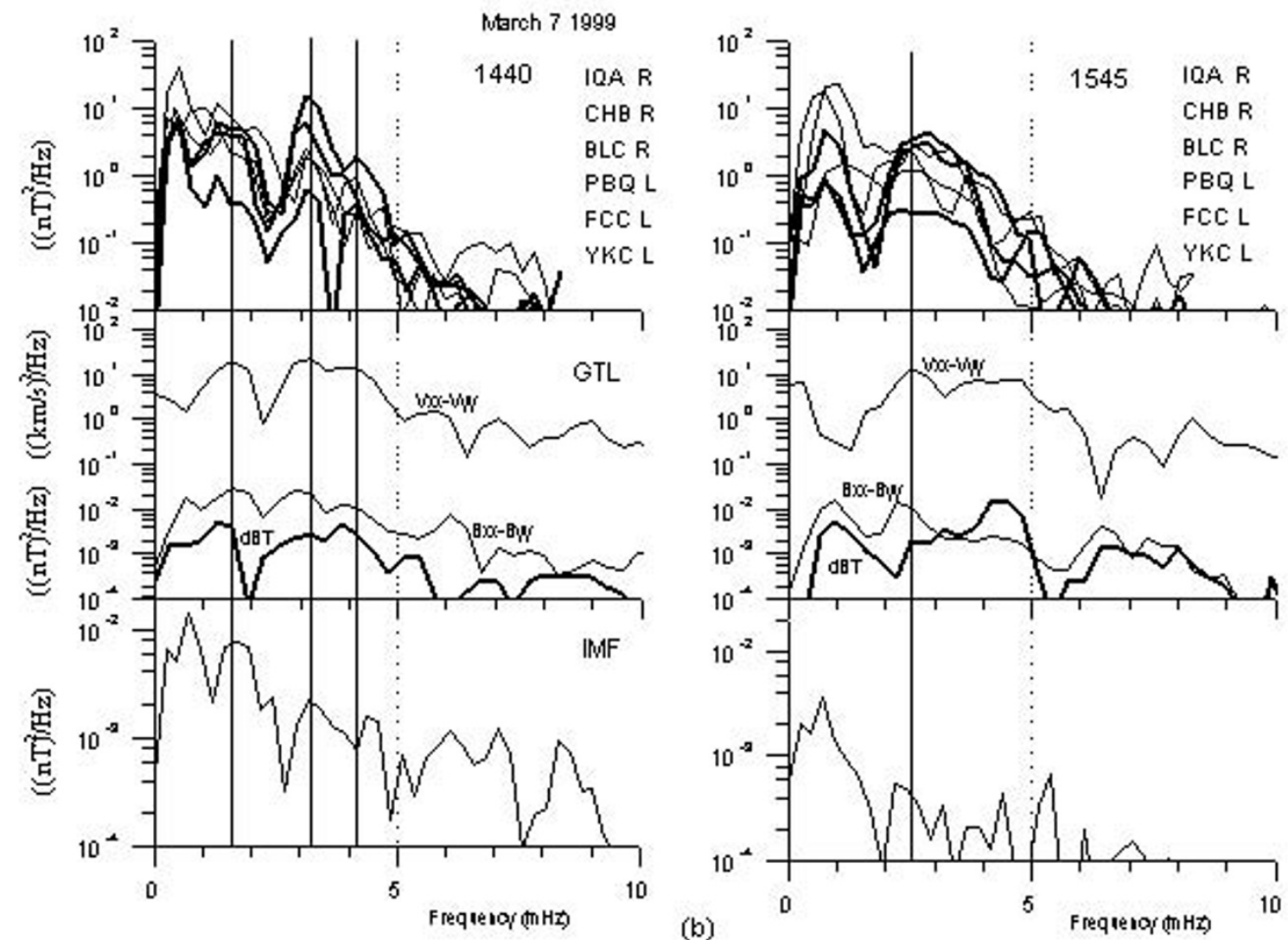

Fig. 8. (a) PSDs for the first wave packet and IMF variations from 13:35 (left panels). Three right panels from the top separately show ground spectra of three groups of stations in the polar cap, in cusp latitudes $\left(\sim 73^{\circ}\right.$ geomagnetic latitude $)$ and in the auroral oval. Spectra plotted in the bottom panel are those of auroral oval stations in the afternoon sector. (b) PSDs for two intervals from 14:40 and 15:45. Top panels present total PSDs of six high latitude stations in the morning sector. Bottom panels shows PSDs of IMF variations. 


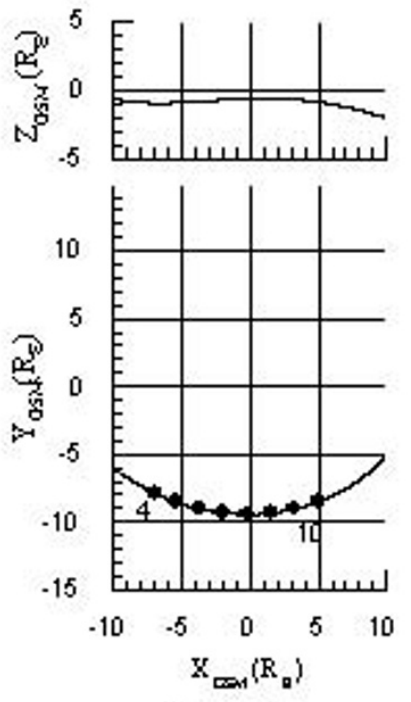

Mny 141999

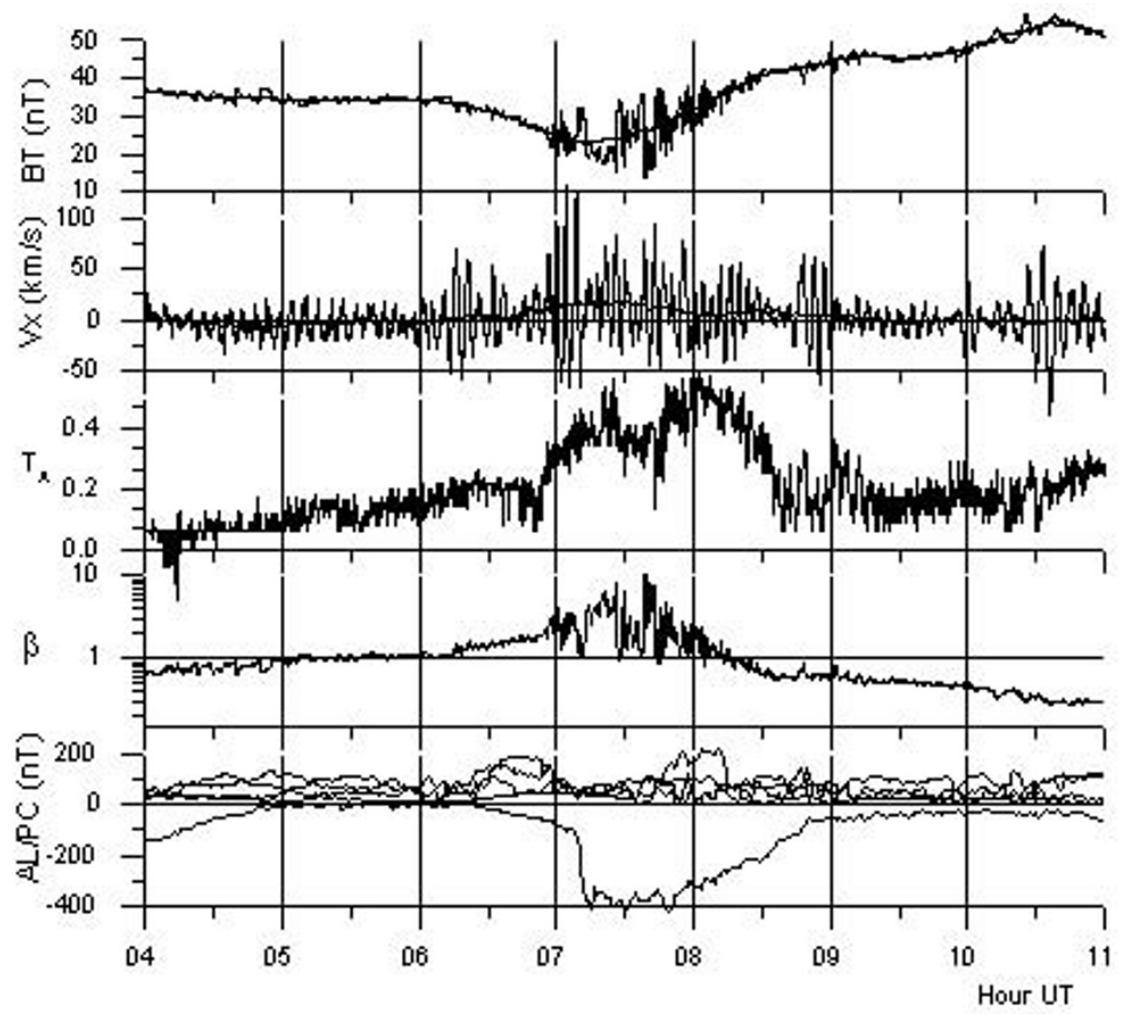

Fig. 9. GTL data and geomagnetic activity on May 14, 1999. Sliding averages of $B_{T}$ and $V_{x}$ are shown in the top two panels. PC indices of four stations, ALE, DVS, HRN and BLC, are plotted in the bottom panel. A decrease of $B_{T}$ from $\sim 6 \mathrm{~h}$ appears to occur associated with the development of substorm.

figuration changes related to the development of substorm.

As for IMF variations, interesting features are seen in spectra shown in Fig. 8. Among them a high correlation between IMF and magnetospheric pulsation spectra is noted in the first and second intervals. Significant peaks are found in the IMF spectra, corresponding to the major peaks of magnetospheric signals at 1.6 and $3.2 \mathrm{mHz}$. However, no good correlation is found in the third interval. The direction of IMF was nearly radial within $\pm 40^{\circ}$ of azimuth in former two intervals. The azimuthal angle of IMF direction became off radial from $\sim 340^{\circ}$ and IMF PSD significantly decreased in the third interval.

\subsection{May 14, 1999}

Next, we examine an event in which a drastic change of the wave characteristics was observed in association with the expansion phase onset of substorm. In Fig. 9 are illustrated variations of field magnitude, plasma flow, plasma $\beta$ and temperature anisotropy along with the ground substorm activity on May 14, 1999. Pc 5 waves were observed throughout the seven hour interval in the dawn flank of $L \sim 10$. Monochromatic wave packets of the L-mode with a frequency of $3.2 \mathrm{mHz}$ appeared without a significant compressional component during 2.5 hour interval from 4:20. $\mathrm{AL}$ and $\mathrm{PC}$ indices indicate that the substorm growth phase began around 6:20 and the onset of expansion phase occurred around 7:00. In association with the development of substorm expansion phase the wave activity suddenly changed with a field magnitude increase. The plasma $\beta$ and the temperature anisotropy also increased, namely, the perpendicular temperature became larger than the parallel temperature. The change in the background plasma condition appears to result in a change of wave characteristics in the magnetosphere. It is evident that these increases of $\beta, T_{A}$ and the field magnitude gradually decayed with the recovery of substorm.

Figure 10 shows variations of magnetic field and plasma velocity along with ground magnetic variations. Significant changes in the magnetic variation at GTL are clearly noted. Amplitudes of waves enlarged after the expansion phase onset around 6:50 both in the magnetosphere and on the ground. Especially, higher frequency oscillations are noted in the compressional component at GTL, while such a change is not so clear in the time-amplitude records of the transverse flow and the ground magnetic variation. It is also evident that the wave frequency became higher during substorm. Normalized amplitudes of the compressional fluctuation attained to more than 0.4 in this case. From the regression analysis for the interval of 6:55-8:10, we obtain correlation coefficient of 0.88 and regression line with a slope of 0.65 between field magnitude variations and those estimated from pressure variations. This suggests that the wave property changed to be compressional and of high $m$ number in association with the occurrence of substorm. Lower frequency transverse waves with a small compressional component followed the substorm recovery after 9:40, as seen in Fig. 9.

The change in wave property associated with substorm is evident from the spectrum analysis (Fig. 11). A wave packet from 6:00 before the substorm occurrence has a peak spectrum at $3.2 \mathrm{mHz}$, and the spectral density of the compres- 


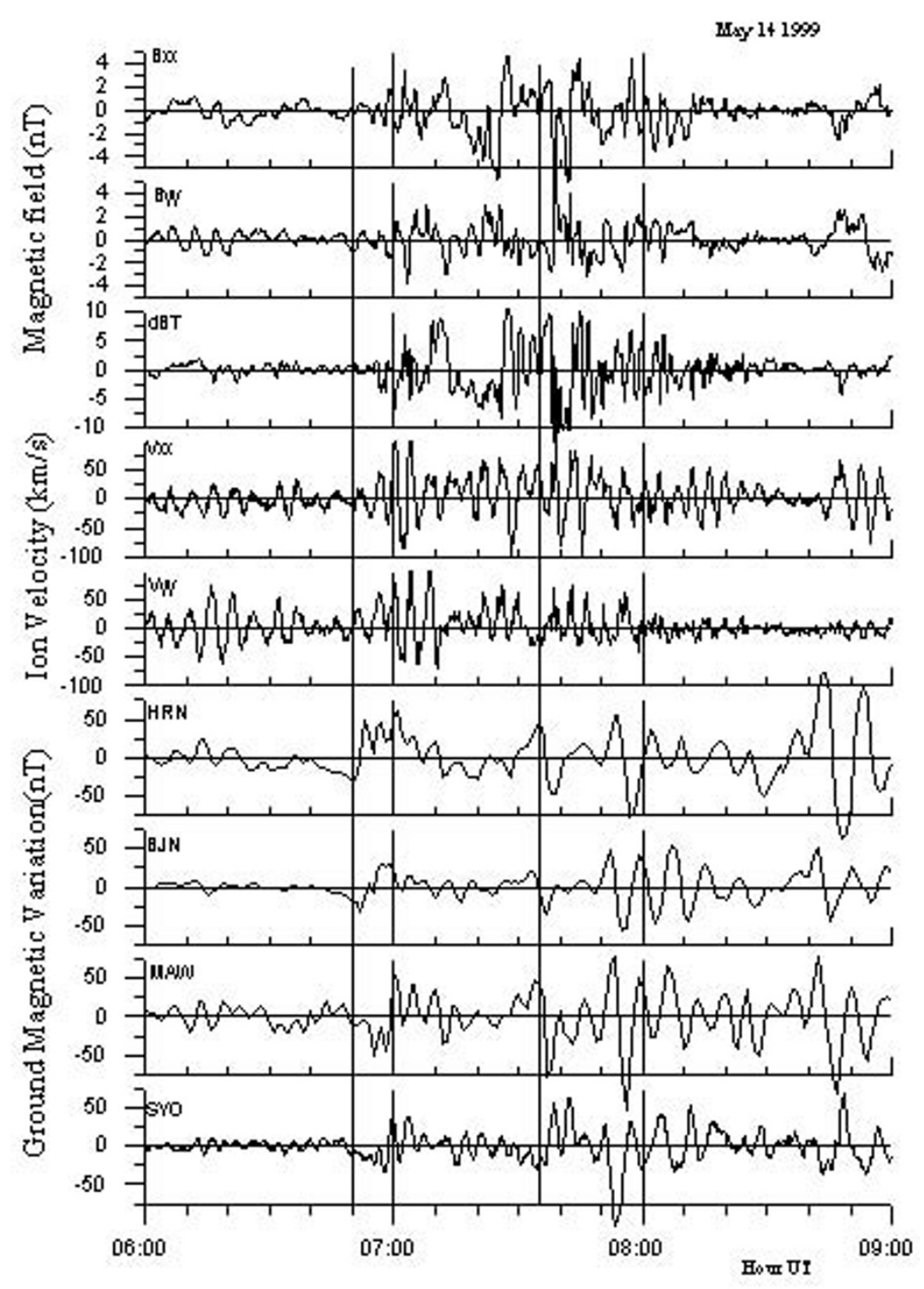

Fig. 10. Magnetic field and plasma flow variations on GTL and high-pass filtered records from ground stations (HRN, BJN, MAW, and SYO) for the three hour period, corresponding to substorm.

sional signal was one order of less than that of the transverse signal. Spectra of two intervals from 6:50 and 7:35 after the expansion phase onset are complicated, as shown in Fig. 11(b) and (c). The spectral peak in plasma velocity shifted to higher frequencies, 3.8 and $4.2 \mathrm{mHz}$. However, these frequency peaks are not found in spectra of the compressional component. Ground signals corresponding to the major peak of $4.2 \mathrm{mHz}$ are not observed in the last interval, though strong signals corresponding to the sub-peak at $2.2 \mathrm{mhz}$ are detected in the ground spectra. The spectral density of compressional component during substorm increased more than one-order of magnitude as compared with the previous level in the Pc 5 frequency range. The increase of spectral density in the last interval are notable in the frequency range above $5 \mathrm{mHz}$. However, ground signals corresponding to these components are not obvious. This is probably because waves during substorm changed to be of compressional nature with a high $m$ number. The peak frequency shift and the spectral enhancement in higher frequencies are features common in substorm-associated wave events as observed in the previous example. As for the relation to IMF variations, we could not find any definite correspondence between IMF and magnetospheric spectra in the last interval.

Ziesolleck and McDiarmid (1995) have presented that the $K_{\mathrm{p}}$ dependence of Pc 5 occurrence and power distributions indicated a shift in the Pc 5 occurrence distribution toward higher frequencies with $K_{\mathrm{p}}$ and that the power increases with increasing $K_{\mathrm{p}}$ almost independent of frequency. Frequency shift toward higher frequency with the development of substorm as observed in May 14 event and in cases examined previously, is consistent with their result. The power increase is dominantly observed in the compressional component in the outer magnetosphere. A similar enhancement of compressional signal could be identified in other wave events which occurred during substorm activity. It is found 


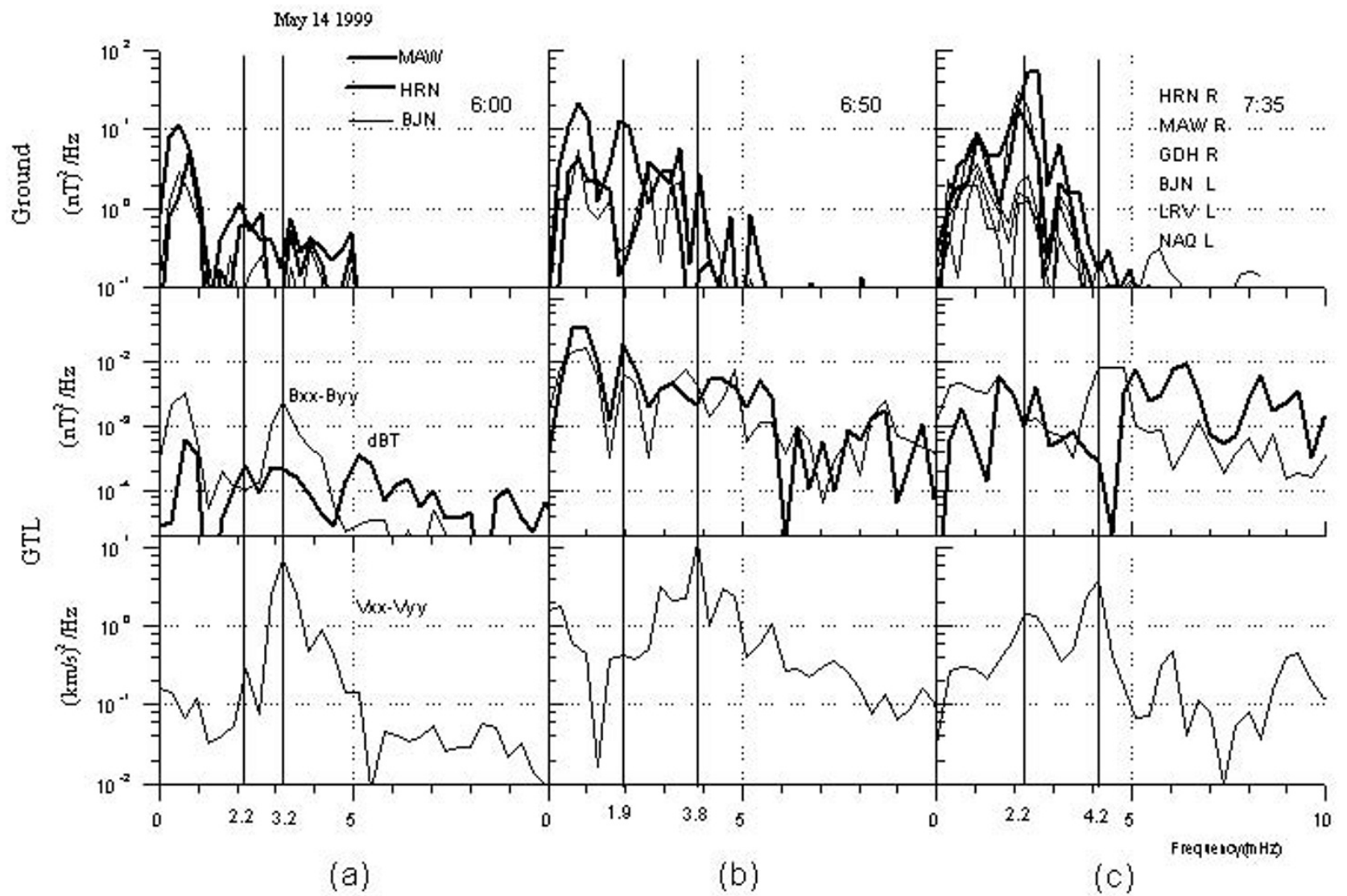

Fig. 11. Comparison of PSDs among the three intervals from 6:00, 6:50 and 7:35. The first interval corresponds to the growth phase. The second and third intervals correspond to the expansion phase and recovery phase of substorm, respectively. PSDs of magnetic variations at three stations, HRN, MAW and BJN, are shown in top panels of (a) and (b). PSDs at three stations LRV, GDH and NAQ, are added to the top panel of (c).

that the occurrence of compressional waves occasionally follows the disappearance of transverse waves associated with the substorm development.

\subsection{August 20, 1999}

Wave events previously examined are cases observed on the morning side, in which a series of wave trains appeared during several hours. Such a series of wave trains with a long duration is rarely observed in the afternoon to evening sector. Most of waves are isolated, of which duration is approximately one hour. Figure 12 shows two examples of isolated wave packets detected on the dusk side. It is worth mentioning here that the background plasma flow is sunward in these cases and the $\beta$ value is larger than 1 on the dusk side.

A flow oscillation of a damped-type waveform began around 17:23 on August 20, 1999. The geomagnetic foot point of GTL was located at latitude of about 73 degree in the meridian through Iceland, according to the orbit data available from the DART database. A similar damped type form of variation was observed at TJO in Iceland. The frequency of the major spectral peak is $2.1 \mathrm{mHz}$, and the wave polarization is right-handed. A sub-peak appears around 3.8 mHz (Fig. 13(b)).

The IMF data shown in Fig. 13(a) suggest that this wave was excited by a sudden rotation of IMF. The $B_{x}$ component of IMF suddenly changed sunward in association with a southward turning of $B_{z}$ around 1712. The solar wind speed was as high as $\sim 600 \mathrm{~km} / \mathrm{s}$. A small increase of 50 $\mathrm{km} / \mathrm{s}$ occurred with the rotation of IMF, but the dynamic pressure was almost constant around $1 \mathrm{nPa}$. On the ground a large impulsive variation of $\sim 200 \mathrm{nT}$ was recorded at IQA in cusp latitude near noon. Amplitudes of the initial pulse at other stations were less than $100 \mathrm{nT}$. This localized large impulse in the northern cusp region near noon would be produced by the reconnection associated with a sudden IMF rotation. The reversal of $B_{x}$ associated with a simultaneous southward turning of $B_{z}$ is a favorable condition for the reconnection in the northern cusp region. In fact, magnitudes of initial impulses were confirmed to be much smaller in the southern polar cap.

Pulsative variations with frequency of $2.1 \mathrm{mHz}$ were observed at stations, PBQ, NAQ, LRV, TJO, and SYO, which are situated along the auroral oval on the afternoon to dusk side. The longitudinal scale of this oscillation is estimated to be approximately 105 degrees in the local time sector of 11-18 hour.

Ground spectra shown in Fig. 13(b) indicate that spectral peaks corresponding to the GTL spectrum are evident in the auroral oval, while lower frequency components dominate in higher latitudes. The polarization at auroral oval stations was right-handed, same as that at GTL, while the polarization at BLC, IQA and GDH was left-handed. This polarization reversal around cusp latitudes means the signature of field line resonance. It is also noted that there exist dif- 


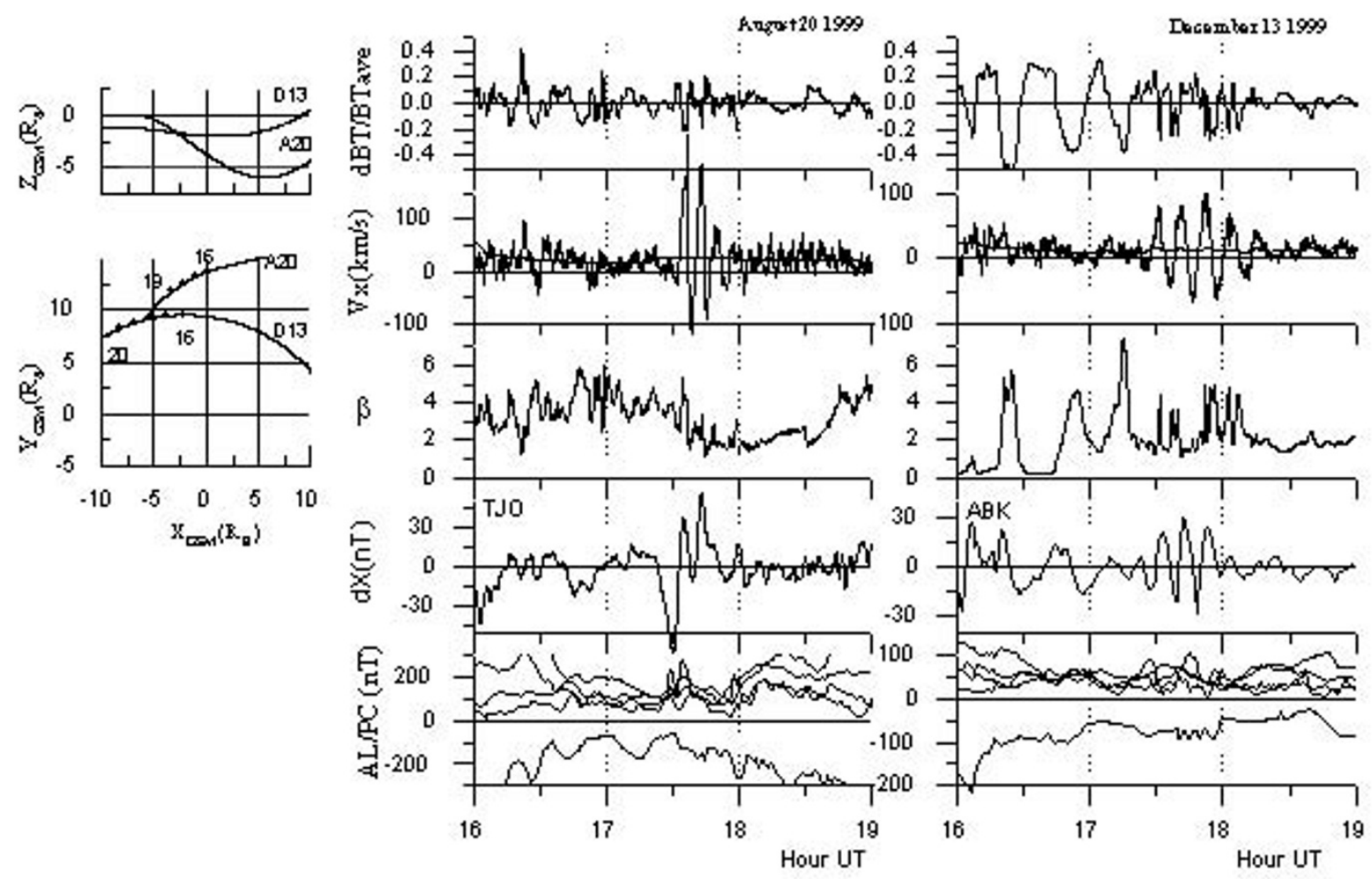

Fig. 12. GTL data obtained on the dusk side and ground magnetic activity on August 20 and December 13, 1999. PC indices of four stations, RES, IQA, BLC and CBB, and of DRV, DVS, IQA and BLC are depicted for August 20 and December 13, respectively.

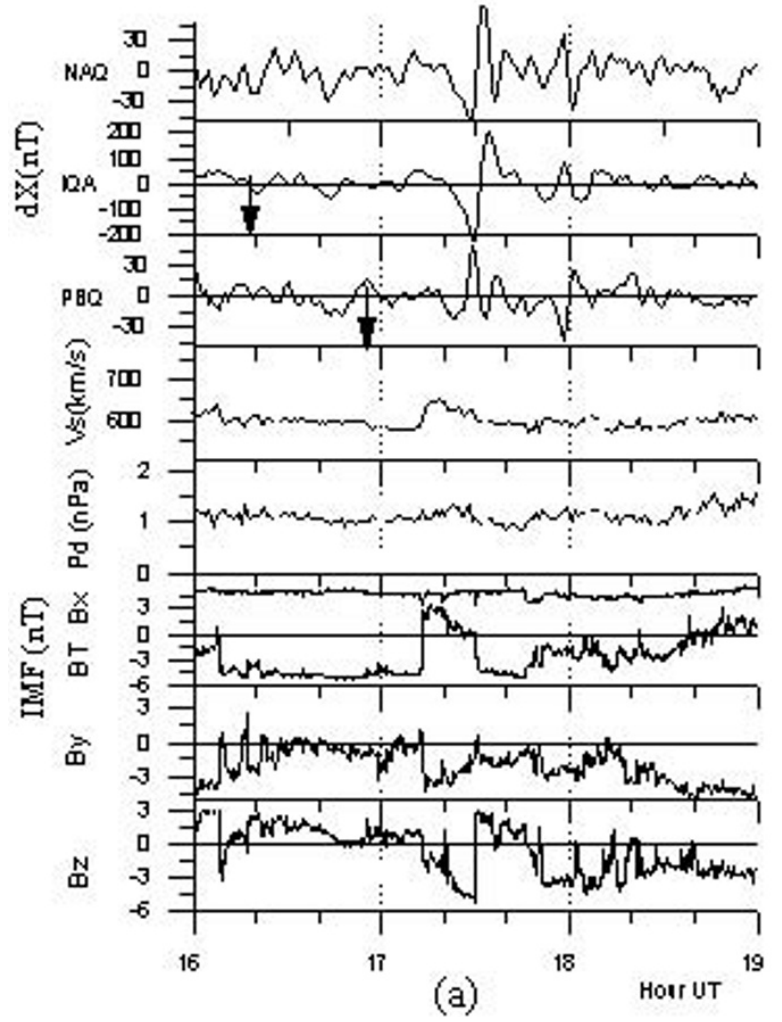

(a)

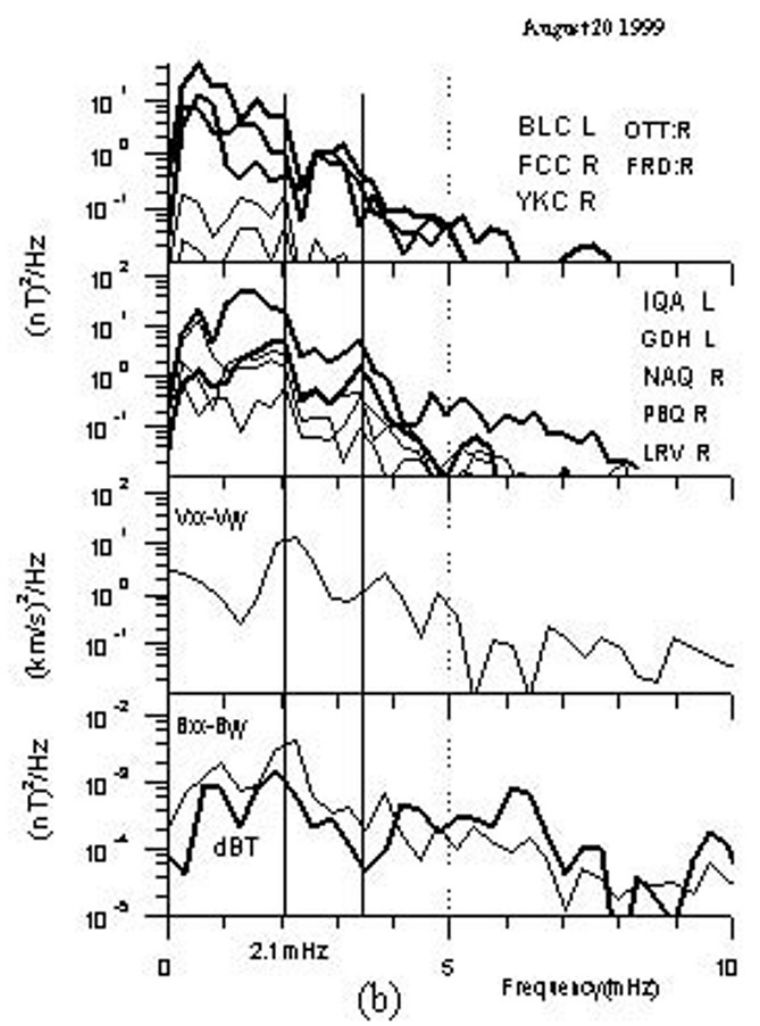

(b)

Fig. 13. (a) High-pass filtered records from high latitude stations, NAQ, IQA and PBQ, and solar wind and IMF variations observed by the ACE spacecraft (time shifted by $41.5 \mathrm{~min}$ ) for the three hour interval from $16 \mathrm{~h}$. The arrows indicate magnetic local noon at IQA and PBQ. (b) Comparison of ground and GTL PSDs in the interval from 17:20. Ground spectra are shown for the morning (top panel) and afternoon sides (second panel from the top), respectively. 


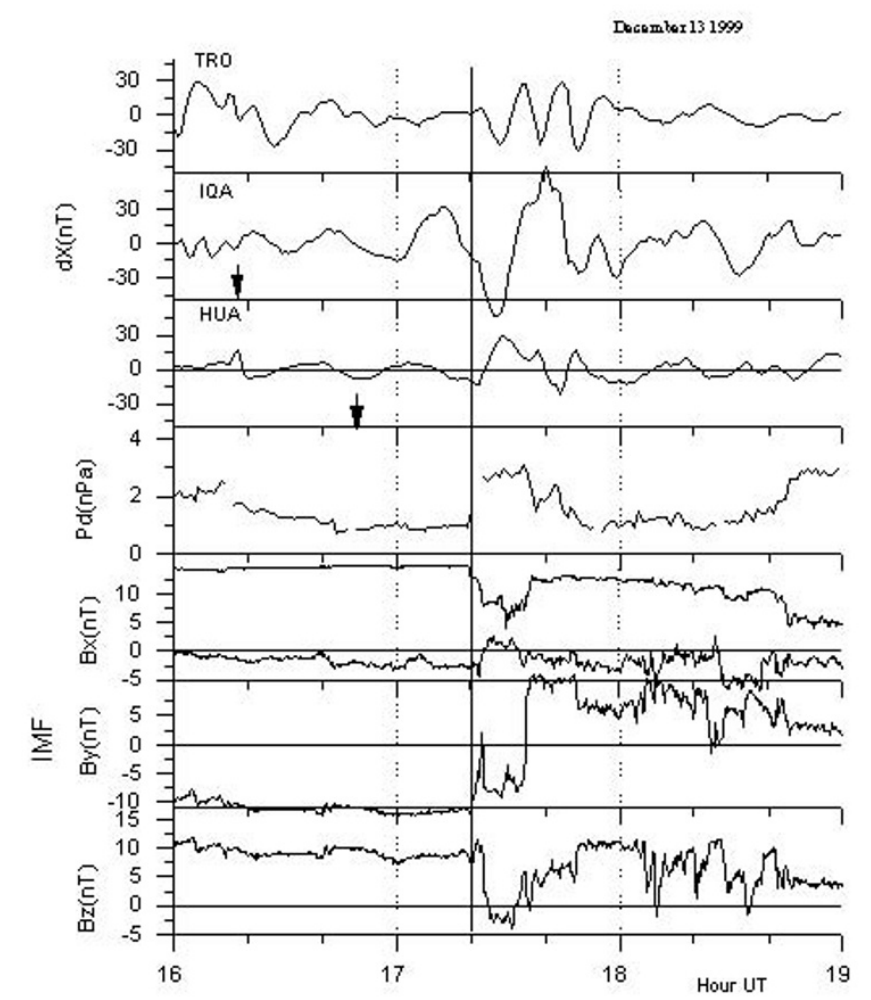

(a)

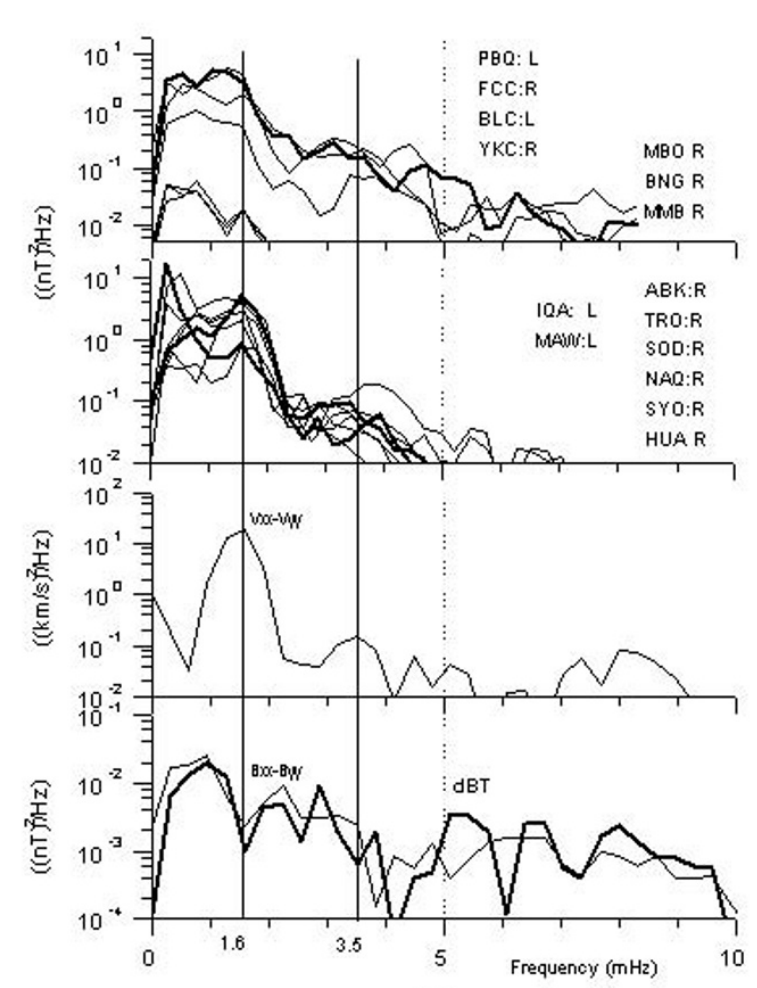

(b)

Fig. 14. (a) Geomagnetic variations at TRO, IQA and HUA, solar wind and IMF variations observed by the ACE spacecraft (time shifted by 53.5 min) on December 13, 1999. The arrows indicate magnetic local noon at IQA and HUA. (b) Comparison of ground and GTL PSDs in the interval from 17:25. The top panel shows ground PSDs at high latitudes in the forenoon region. PSDs at three low latitude stations, MBO, BNG and MMB are shown in the left bottom part. The second panel shows PSDs at high latitude stations and HUA in the dip equator in the afternoon sector.

ferences in the spectral feature between the afternoon and forenoon sectors. A sharp peak of spectra at $3.4 \mathrm{mHz}$ appears on the afternoon side, whereas a broad peak at 2.9 $\mathrm{mHz}$ is apparent on the forenoon side. Spectra at stations in mid-latitudes on the day side (OTT and FRD), as shown thin lines in the top panel of Fig. 13(b), are very similar to those at high latitude stations in the morning to noon sector. These differences appear to be attributable to regional differences of the magnetospheric structure to produce the field line resonance. As for IMF changes, multiple spectral peaks at 1.7, 2.1, 2.7 and $3.7 \mathrm{mHz}$ were noted, which roughly correspond to those of magnetospheric oscillations.

Villante et al. (2006) have presented a case in which power enhancements (at 1.4, 2.5, and $4.2 \mathrm{mHz}$ ) simultaneously occurred in the $H$ component on the low latitude ground as well as in the radial component of the SW speed and IMF, while the SW density showed minor enhancements at different frequencies. This observation is essentially similar to the case discussed above. They have speculated that Alfvénic fluctuations of IMF as possible drivers of geomagnetic field fluctuations at discrete frequencies.

\subsection{December 13, 1999}

The wave event on December 13, 1999 was associated with an increase of $1.5 \mathrm{nPa}$ in the solar wind ram pressure. The $B_{y}$ component of IMF suddenly changed duskward in association with a southward turning of $B_{z}$ around 17:20 (Fig. 14(a)). The solar wind speed was in range of 450-480 $\mathrm{km} / \mathrm{s}$. This dynamic pressure increase and IMF variations caused a sudden impulse of $\sim 5 \mathrm{nT}$ in low and middle latitudes and an equatorial enhancement of $40 \mathrm{nT}$ in the horizontal component at Huancayo.

In high latitudes periodic variations with the frequency of $1.6 \mathrm{mHz}$ and the right-handed polarization were observed in a wide longitudinal sector of $\sim 145$ degrees (local time range of 9-18 hour). Largest spectral peaks were found at ABK and TRO near the conjugate region of GTL on the dusk side (Fig. 14(b)). PSDs in the dayside cusp latitude (IQA and BLC) have large left-handed components in the frequency range below $1.6 \mathrm{mHz}$. The polarization is also left-handed in the afternoon cusp latitudes (GDH, MAW and $\mathrm{BJN}$ ). This indicates that the polarization reversal occurs around the polar cap boundary in a wide longitudinal sector. A common peak was observed at frequency of 3.5 $\mathrm{mHz}$ in both the ground and GTL spectra, though the peak power is small as compared with that at the main peak. In middle and low latitudes the spectral peak at $1.6 \mathrm{mHz}$ was a common feature in wide longitudes, but the $3.5 \mathrm{mHz}$ peak was not detected in the forenoon region. Spectra at low latitude stations, $\mathrm{MBO}, \mathrm{BNG}$ and $\mathrm{MMB}$ are shown by thin lines in the top panel of Fig. 14(b). The $1.6 \mathrm{mHz}$ peak intensity at HUA (the second panel of Fig. 14(b)) is approximately 40 times more than those at low latitude stations. The $1.6 \mathrm{mHz}$ periodicity was found from the spectral analysis of both solar wind density and IMF fluctuations after the sudden increase.

This equatorial enhancement of sudden impulse is proba- 
bly caused by the electric field produced by transmission of the induced electric field in the polar ionosphere by southward IMF changes (Kikuchi et al., 1996). Kikuchi et al. (2000) have also found that an impulsive magnetic change with a timescale of 100 seconds observed in the polar region appears at the dayside dip equator with a time delay of about 10 seconds and with an enhancement of factor 3 as compared with amplitudes at middle latitudes. An enhancement factor of 8 was observed in the present case.

Shimazu et al. (1995) reported the analysis of a global response of ground magnetic variations associated with solar wind dynamic pressure increase. Their event occurred under a solar wind condition of stable northward IMF direction (almost tangential to the magnetopause), and high solar wind speed $(620 \mathrm{~km} / \mathrm{sec})$. December 13 event was not associated with large solar wind disturbances as the event examined by Shimazu et al. (1995) and other events examined by Villante et al. (2007). Geomagnetic responses in low latitudes are essentially similar to events analyzed by them. A global magnetospheric oscillation with the frequency 1.6 $\mathrm{mHz}$ was probably excited by the combined action of solar wind density and IMF variations following the sudden increase. Rostoker and Sullivan (1987) reported that a large fraction of the afternoon pulsations they observed were perhaps driven by impulses in the solar wind rather than the magnetopause instabilities. However, a few cases, which were associated with impulses in the solar wind, were found in the present analysis.

A direct driven process by solar wind density fluctuation, as suggested by Kepko and Spence (2003), would not fully account for magnetospheric responses without consideration of the internal resonance process of the magnetosphere, since magnetospheric responses to solar wind fluctuations have a dawn-dusk asymmetry, as observed in December 13 case. Villante et al. (2007) suggests that geomagnetic fluctuations can be driven directly by fluctuations of the solar wind density at the same frequencies. On the other hand, the repeated occurrence in the same frequency bands and some evidence for amplification processes suggest additional contributions possibly related with cavity/waveguide resonances.

The common feature of two events examined above is that magnetospheric oscillations occurred over a wider range of local time in response to solar wind discontinuities and/or dynamic pressure increases and that largest variations on the ground were observed in the dayside cusp region. Waveforms were less regular in the forenoon sector than in the afternoon sector.

\section{Statistical Result}

A total of 330 wave packets in the Pc 5 range were identified from the survey of six year GTL data. Wave events were selected on the basis of amplitudes of more than approximately $30 \mathrm{~km} / \mathrm{s}$ in the plasma velocity by the visual inspection of time amplitude records of plasma flow and magnetic field. Normalized amplitudes of the compressional component are mostly less than 0.1 in the selected samples. The complex FFT analysis was applied to both plasma flow and magnetic field data. In the following statistics the plasma flow data were mainly used to examine the frequency and polarization, since the spectrum of magnetic field variations detected by an equatorial spacecraft does not necessarily coincide with that of flow oscillations. This is because that FLR waves of the fundamental mode have the node in the equatorial plane. The wave spectrum has not always a single peak, and has often two or more peaks, as shown in the event analysis. Therefore the statistics was made for the frequency and polarization of the major spectral peak.

Figure 15(a) represents the occurrence histogram of wave frequency plotted separately for events observed on the forenoon and afternoon sides. The present statistics show that a number of preferential frequencies in the morning sector occur centered around 1.9, 2.5, and $3.3 \mathrm{mHz}$ and also possibly $4.5 \mathrm{mHz}$ in the outer magnetosphere, while the dominant frequency is not obvious in the afternoon sector. These dominant frequencies essentially agree with those observed on the ground, though a preferential frequency at $1.3 \mathrm{mHz}$ is not evident in the present statistics. Ground observations have indicated that the existence of multiple, discrete spectral lines of stable frequencies of 1.3, 1.9, 2.6 and $3.4 \mathrm{mHz}$ (Samson et al., 1992b; Ziesolleck and McDiarmid, 1994, 1995; Chisham and Orr, 1997). Francia and Villante (1997) examined spectra from the low-latitude L'Aquilla $(L=1.6)$ ground station and suggests that the oscillations occur over bandwidths of $0.2 \mathrm{mHz}$ such that $f=1.2-1.4,1.8-2.0,2.4-2.6$, and 3.2-3.4 mHz. More recently, Viall et al. (2009) find that certain frequencies, specifically $f=1.0,1.5,1.9,2.8,3.3$, and $4.4 \mathrm{mHz}$, occur more often than do other frequencies in 10 years (19962005) of dayside magnetospheric data at synchronous orbit. Plaschke et al. (2009b) found in a statistical survey of magnetopause undulation and motion characteristics based on the THEMIS measurements that the magnetopause oscillations showed characteristic frequencies, which coincide with a set of stable and recurrent frequencies, mentioned above.

Figure $15(b)$ is the distribution of the L-mode and Rmode polarizations for observed locations. The L-mode waves dominate in the dawn sector while the R-mode waves are mostly observed on the dusk sector. The occurrence rate of left-handed and linear polarizations is $87 \%$ in the morning sector, whereas that of right-handed and linear polarizations is $82 \%$ in the afternoon sector. It is interesting to note here that the L-mode predominates before local time of about 11 hour, though wave events were a few near noon. This suggests that the polarization distribution is not symmetric to the noon meridian. The distribution of polarization in the outer magnetosphere is consistent with the dependence of polarization on the local time of observations revealed in early studies on ground data (Kokubun and Nagata, 1965; Samson et al., 1971; Samson and Rostoker, 1972; Chisham and Orr, 1997). As already shown in previous studies (e.g., Kokubun et al., 1989; Anderson et al., 1990; Nosé et al., 1995), the local time asymmetry of wave occurrence is also evident in Fig. 15(c).

Junginger et al. (1985) statistically investigated Poynting vectors of Pc 5 pulsations observed at synchronous orbit by the GEOS-2 satellite. They found that the Poynting fluxes measured were in the range from $10^{-10}$ to $10^{-5}$ 


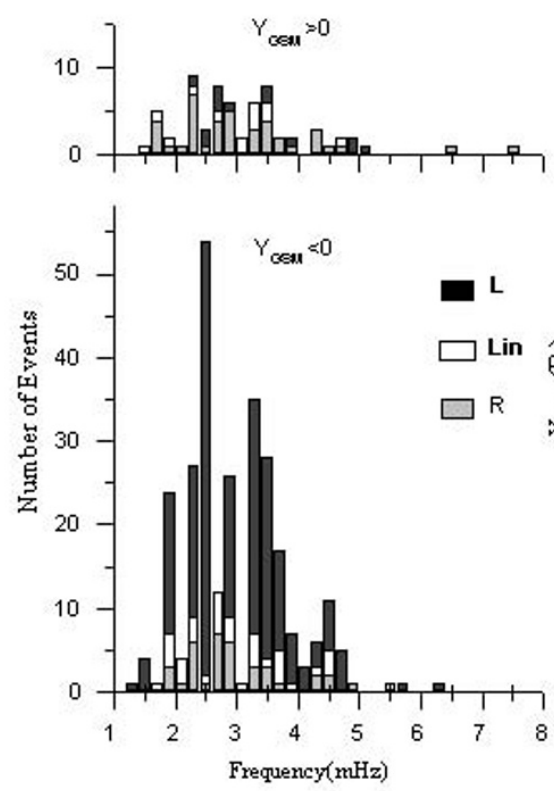

(a)

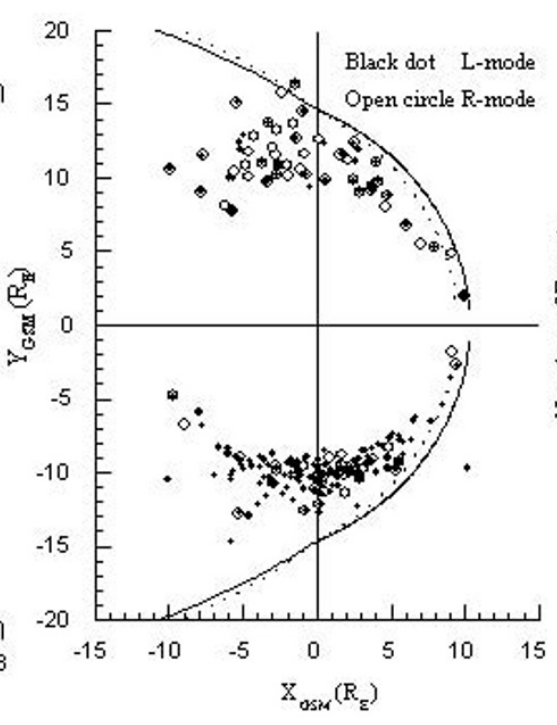

(b)

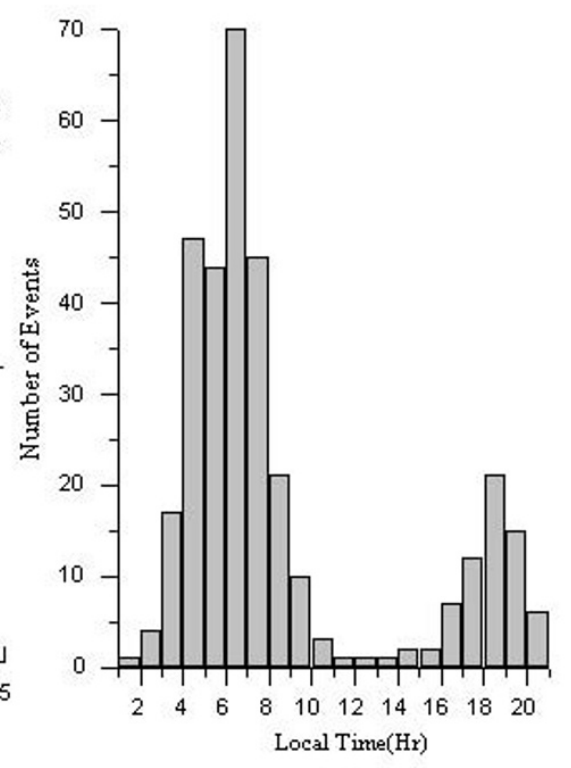

(c)

Fig. 15. Occurrence histogram of wave frequency (a), distribution of wave polarization (b), and local time distribution of wave occurrence (c).

$\mathrm{W} / \mathrm{m}^{2}$. Most of the fluxes lay in the range from $10^{-9}$ to $10^{-7}$ $\mathrm{W} / \mathrm{m}^{2}$. Poynting fluxes associated with transverse waves in the outer magnetosphere have already been discussed by using GTL electric field data (Sakurai et al., 2001). They observed tailward propagation for 12 cases in the dawn sector and only one case is radially outward. In the afternoon and dusk sectors each case is tailward. The Poynting flux of transverse waves was further examined in applying plasma flow data. An example of time variation of both field-aligned and perpendicular directions of Poynting flux has been already shown in Fig. 2. The Poynting flux of Pc 5 waves was calculated for most of selected events. However, a stable direction of the azimuthal vector of Poynting flux for all of selected events could not be determined. This would be partly because of overlapped and irregular or impulsive disturbances of different origins or a less coupling with the compressional component in some cases. As a result, stable directions of the Poynting vector in the plane perpendicular to the magnetic field were determined for about a half of the selected events.

The energy flow vectors are plotted in the left panel of Fig. 16. Most of the Poynting fluxes are anti-sunward with a small inward component. Out of 165 events 22 events have sunward component as shown in the right bottom panel of Fig. 16. A strong contrast between the morning and evening sides is evident in the distribution of the fluxes. The distribution of flux magnitudes is shown in the right upper panel of Fig. 16. The median values of the dawn/dusk distribution are 1.6 and $0.5 \mu \mathrm{W} / \mathrm{m}^{2}$, respectively. The flux is smaller in the dusk sector, as similar to the previous results observed at the synchronous orbit (Junginger et al., 1985).

As has been shown in the event analysis, regular flow oscillations generally occur under a low $\beta$ condition around 1 , especially in the morning sector. However, large $\beta$ val- ues are often observed corresponding to wave events during substorm and isolated wave events on the dusk side. To confirm these feature further the averages of plasma $\beta$ and sunward flow at times of the wave events were statistically examined. The hourly averages of $V_{x}$ and $\beta$ are considered as measures to represent the background plasma state. In these statistics hourly averages were obtained as the 301 (3672 s) point sliding average. Figure 17 shows the occurrence histograms of $V_{x \mathrm{av}}$ and $\beta_{\mathrm{av}}$ at times of wave events for both transverse and compressional waves. Though the detail of the analysis is not given here for compressional waves observed with GTL, the histograms of $V_{x \mathrm{av}}$ and $\beta_{\mathrm{av}}$ at times of the compressional wave occurrence are shown for comparison. Compressional waves were selected in taking into account the antiphase relation between plasma and magnetic pressures, and the normalized amplitude of field magnitude. The histograms for compressional waves are shown in the top two panels of this figure.

The background state of plasma at times of wave occurrence is significantly different between the forenoon and afternoon sectors. It is noted that the background plasma flow is sunward in cases of waves in the evening sector without exception. The flow speed sometimes attains to more than $30 \mathrm{~km} / \mathrm{s}$.

Transverse waves are generally observed in the condition of $\beta$ below 1.5. High $\beta$ cases are mostly associated with events on the dusk side. As for compressional waves, background plasma flow and $\beta$ conditions are significantly different from the case of transverse wave. Compressional waves occur under conditions of $\beta$ and flows larger than those of transverse waves.

There are previous works indicating that the occurrence and intensity of Pc 5 pulsations observed near dawn in the equatorial outer magnetosphere are well correlated with in- 

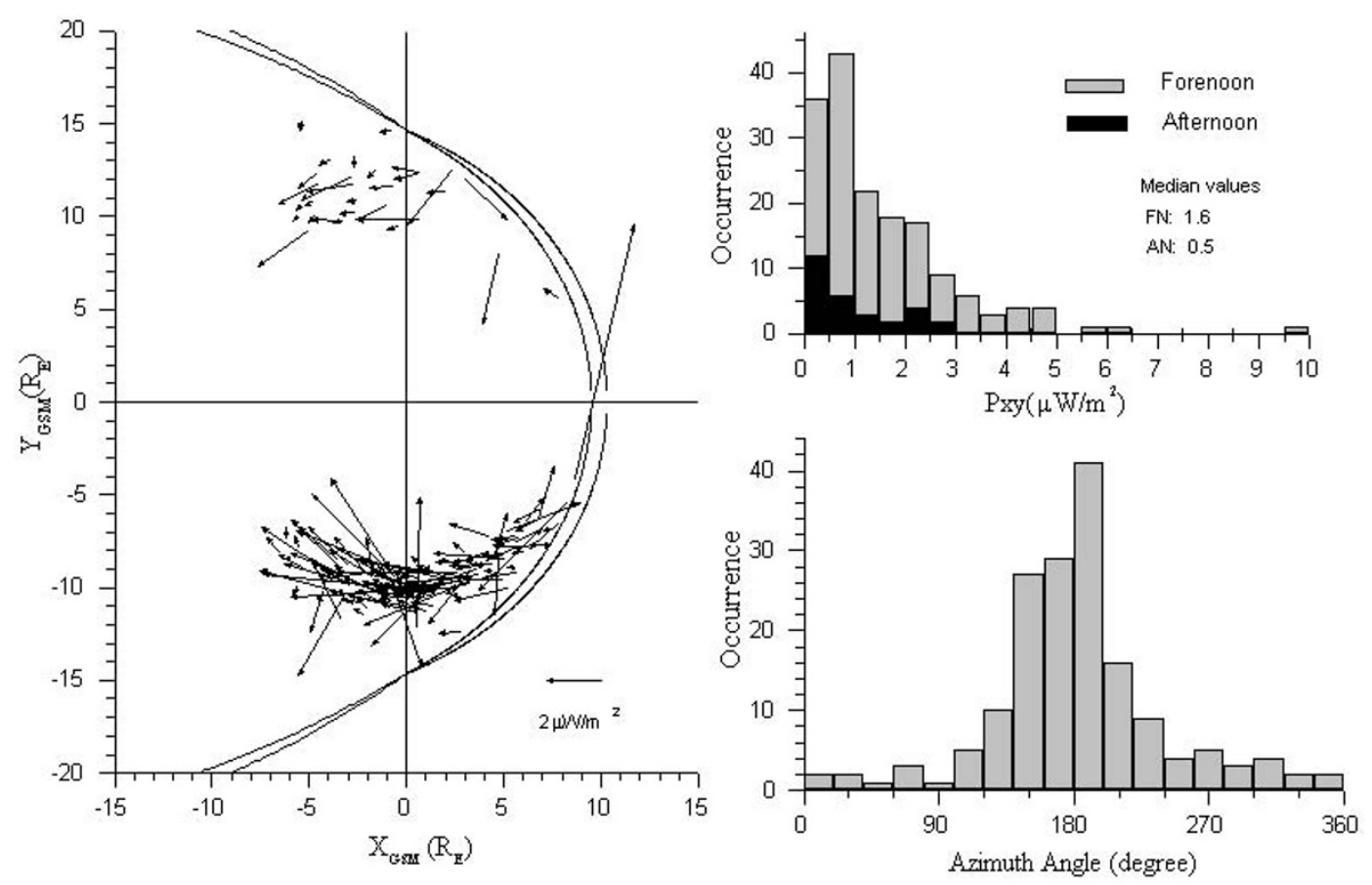

Fig. 16. A summary plot of energy flow vectors in the GSM $X-Y$ plane (left panel). Histograms of azimuthal angles of Poynting flux and the magnitude of Poynting flux are shown in the right panel.
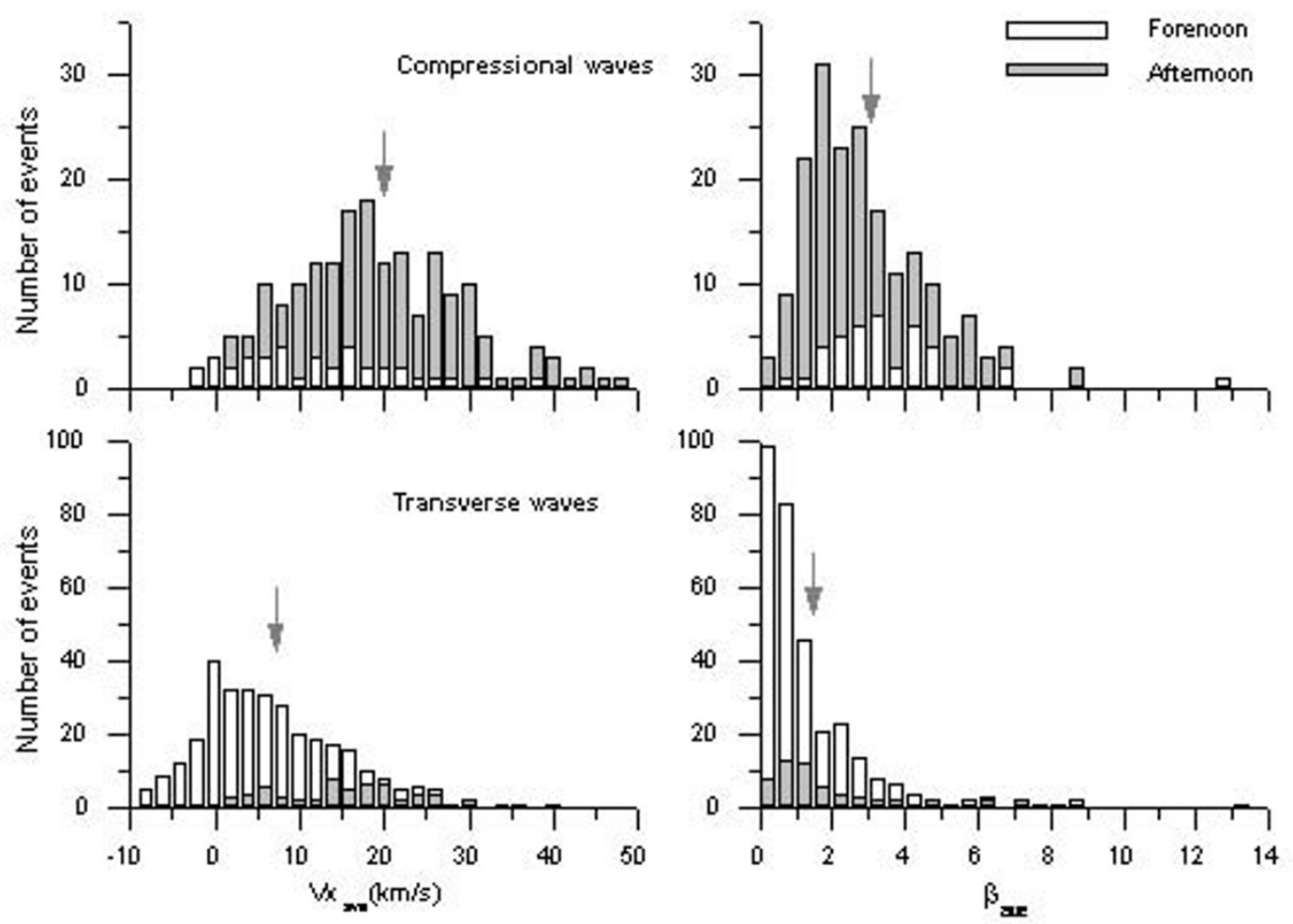

Fig. 17. The occurrence histograms of ULF waves versus the average $V_{x}$ and $\beta$. The histogram for compressional waves are plotted in the top panels for comparison. The arrows indicate the median values of the distributions.

creased solar wind velocity (e.g., Junginger and Baumjohann, 1988; Kokubun et al., 1989). This increased power is commonly attributed to the occurrence of a KHI at the flanks of the magnetopause or at the inner edge of the low- latitude boundary layer (e.g. Miura, 1992; Fujita et al., 1996). Engebretson et al. (1998) and Vennerstrøm (1999) obtained similar correlation values for morning sector Pc 5 band PSD with solar wind speed, and Junginger and 


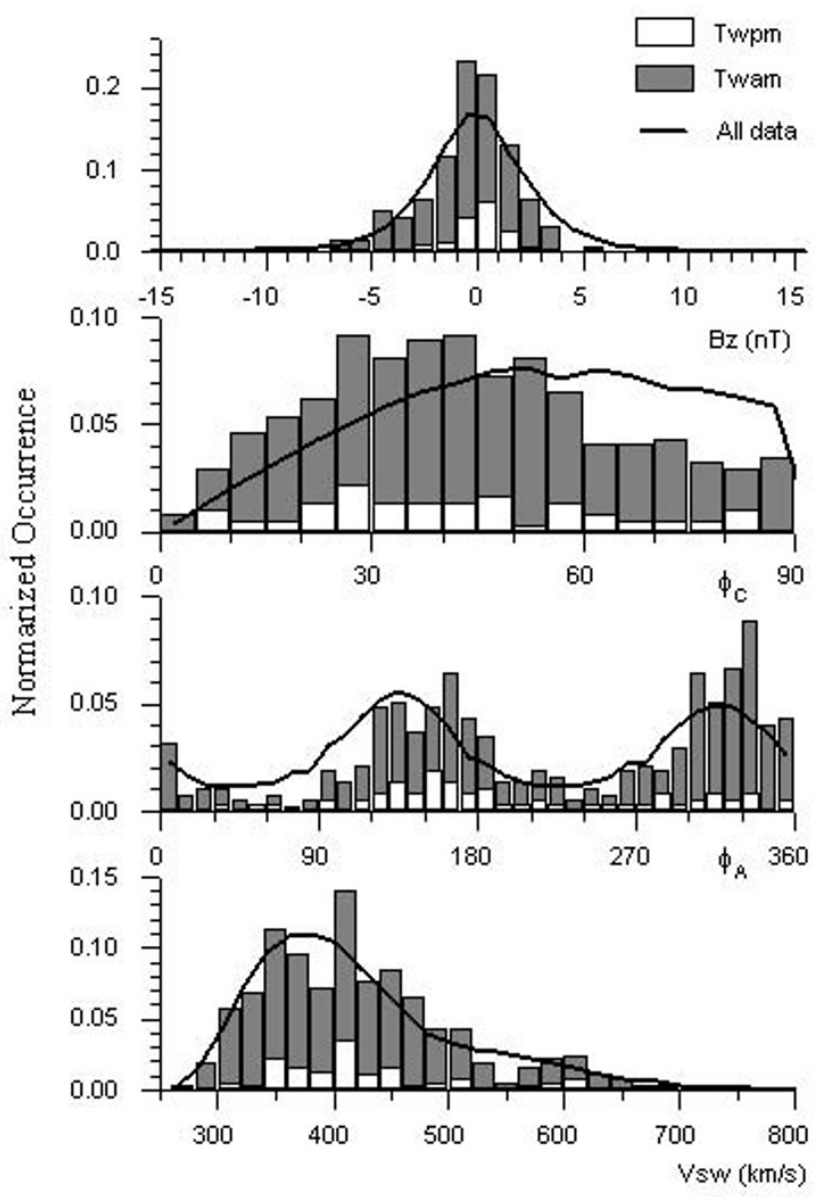

Fig. 18. The normalized occurrences of IMF $B_{z}$, the azimuthal angle $\left(\phi_{A}\right)$ and the cone angle $\left(\phi_{C}\right)$ of IMF, and the solar wind speed $\left(V_{\mathrm{SW}}\right)$, corresponding to transverse waves observed by GTL. The solid lines indicate the normalized occurrences of hourly values for all data sample of solar wind parameters during six year interval of 1995-2000.

Baumjohann (1988) demonstrated the dependence of morning sector pulsation occurrence on solar wind speed. Baker et al. (2003) found that the strong correlation between solar wind speed and Pc 5 band PSD on the ground is independent of local time and that Pc 5 pulsations at all magnetic local times occur preferentially during IMF $B_{z}$ northward conditions. Influences of IMF radial orientation on pulsation amplitudes and occurrence rate have not necessarily clarified in previous studies.

In this study the correlation between the solar wind parameters and the occurrence of Pc 5 waves in the outer magnetosphere were also examined by using OMNI solar wind data (Hourly value) available from CDAWeb at (http://cdaweb.gsfc.nasa.gov/istp_public/). The OMNI data constitute a combination of solar wind measurements from several spacecraft time-shifted to represent the solar wind conditions at the bow shock nose. Figure 18 represents normalized occurrence histograms of events against $B_{z}$, the cone angle (defined as the angle between the Sun-Earth line and the IMF direction) and azimuthal angle of IMF, and the solar wind speed. The solid lines in this figure represent normalized occurrences of hourly values for the all data sample of solar wind parameters during six years of 1995-2000. Histograms denote normalized occurrences of solar wind parameters corresponding to GTL wave events.

As for solar wind speed, the wave occurrence distribution tends to be larger than that of all solar wind data in the range of speed higher than $400 \mathrm{~km} / \mathrm{s}$. This result is not inconsistent with previous studies. Interesting features are that Pc 5 waves tend to occur under the condition of more radial IMF direction than the average spiral angle, and of small $B_{z}$ values and low cone angles. It appears in cases of afternoon events that waves tend to occur during northward IMF. The statistical analysis of the oscillatory motion of the magnetopause based on THEMIS spacecraft observations by Plaschke et al. (2009b) has shown enhanced oscillation activity at a set of preferential frequencies, same as observed on the ground, in the noon local time sector during periods of northward IMF, slow or moderate solar wind speed and low solar wind cone angle. The present statistics show almost same tendency as the result by Plaschke et al. (2009b).

Wave events examined in this statistics were selected basically from plasma flow oscillations with small compressional magnetic field variations. As for afternoon events, we examined the phase relation between flow and magnetic field variations expected from the characteristics of the normal mode FLR. $75 \%$ of events were found to be of FLR type in the phase relation. The definite phase relation was not discernible for remaining $25 \%$ of events, partly because of smallness of magnetic field changes and overlapped irregular variations. Though all of samples do not satisfy the FLR criterion, compressional waves predominating in the afternoon sector, such as studied by Zhu and Kivelson (1991) and Constantinescu et al. (2009), are at least excluded in the present statistics.

\section{Summary}

Large differences in ULF wave characteristics, such as occurrence frequency, polarization, and propagation direction, on either side of noon have been observed before both at high and low latitudes on the ground and in space (e.g., Kokubun, 1985; Kokubun et al., 1989; Anderson et al., 1990; Ziesolleck and McDiarmid, 1994, 1995; Chisham and Orr, 1997; Baker et al., 2003). The characteristics of afternoon sector Pc 5 waves are markedly different from those observed in the morning sector. Satellite observations (Kokubun et al., 1989; Anderson et al., 1990) show that they are predominantly a mixture of compressional and radially polarized waves and that the radially polarized waves have a poor ground correlation. These radially polarized waves are likely to be driven by westward drifting ions from the nightside of the magnetosphere (Southwood, 1976) and will usually have large azimuthal wave numbers $m \sim 100$.

Rostoker and Sullivan (1987) present, in an analysis of the polarization characteristics of Pc 5 pulsations using data from a meridian line of magnetometers straddling the auroral oval in the afternoon and dusk sectors, that afternoon sector Pc 5 pulsations are a rather rare phenomenon compared to their morning sector counterparts. They also show that the latitudinal variation of the polarization characteristics is inconsistent with a simple KHI hypothesis particularly in the local time interval between noon and dusk. They further reported that the afternoon sector events seem 
to have a tendency to occur during episodes of northward interplanetary magnetic field and, in general, appear to be triggered by impulses which have global signatures at times of quiescence or decreasing magnetospheric activity. It is suggested that the afternoon sector Pc 5 waves are the ground signature of compressional waves in the magnetosphere mainly cavity/waveguide modes. They also stated that there is little evidence of field line resonances in the afternoon sector. However, there are reports of FLR observations in the afternoon, though they do occur less often than in the morning. We also confirmed that the polarization reversal in cusp latitudes on the ground were observed for considerable numbers of afternoon events, as in cases discussed in the event analysis.

It is evident from this study that the features of Pc 5 waves observed in the outer magnetosphere differ greatly on either side of noon, in accordance with those observed on the ground. Statistical results are summarized as follows.

1. The L-mode waves dominate in the dawn sector while the R-mode waves are mostly observed on the dusk sector, as already known in the ground-based observations. The occurrence rate of left-handed and linear polarizations is $87 \%$ in the morning sector, whereas that of right-handed and linear polarizations is $82 \%$ in the afternoon sector.

2. Most of the Poynting fluxes are anti-sunward with a small inward component both in the dawn and dusk sectors. This feature is consistent with mechanisms that the excitation energy of waves is fed from the solar wind. The flux is generally smaller in the dusk sector than in the dawn sector.

3. The background state of plasma at times of wave occurrence is significantly different between the forenoon and afternoon sectors. The background plasma flow is sunward in cases of waves in the afternoon sector without exception. The flow speed sometimes attains to more than $30 \mathrm{~km} / \mathrm{s}$ in the dusk sector. Transverse waves are generally observed in the condition of $\beta$ below 1.5. High $\beta$ cases are mostly associated with events on the dusk side.

4. As for solar wind speed, the wave occurrence distribution tends to be larger than that of all solar wind data in the range of speed higher than $400 \mathrm{~km} / \mathrm{s}$. Interesting features are that Pc 5 waves tend to occur under the condition of more radial IMF direction than the average spiral angle, and of small $B_{z}$ values and low cone angles. It appears in cases of afternoon events that waves tend to occur during northward IMF, consistent with ground observations.

Other features to be noted here is that a series of wave trains with a regular wave form often appears during several hours in the forenoon sector. In contrast a series of wave trains with a long duration is rarely observed in the afternoon to evening sector. Most of waves in the afternoon sector are isolated, of which duration is approximately one hour. This difference in wave duration between the forenoon and afternoon sectors would be due to the difference in the background plasma state. High plasma $\beta$ states dominate in the dusk sector, which may result in waves to be of more compressional nature.

Solar wind and IMF conditions were, at first, not taken into account in selection of events for event analysis. We found in analyzing solar wind and IMF data that changes in IMF, especially discontinuities, appears to be related to the onset of wave and changes in wave form both in space and on the ground, as seen in the most of events examined in this study. Therefore, the rotational spectra of magnetic field variations were calculated by the same procedure as the analysis of magnetospheric oscillations in order to examine transverse Alfvénic oscillating features of IMF and to compare them with wave spectra. It is found that a good correspondence is often observed between spectral components of magnetospheric and IMF fluctuations. Though it is needed to further examine conditions to relate IMF fluctuations to magnetospheric variations, it is suggested that IMF Alfvénic fluctuations is an important source for the excitation of magnetospheric waves. Villante et al. (2006) also speculated that Alfvénic fluctuations as possible drivers of geomagnetic field fluctuations at discrete frequencies.

It was found from the event analysis that the spectral density of compressional component during substorm in space was enhanced more than one-order of magnitude as compared with the previous level in the Pc 5 frequency range. The increase of spectral density is notable in the frequency range above $5 \mathrm{mHz}$. It was also noted that waves during substorm changed to be of compressional nature probably with a high $m$ number. The peak frequency shift and the spectral enhancement in higher frequencies are features common in substorm-associated wave event. Ziesolleck and McDiarmid (1995) have shown that the low frequencies (1-2 $\mathrm{m} \mathrm{Hz}$ ) had a relatively high occurrence for low geomagnetic activity levels $\left(K_{\mathrm{p}}<2\right)$, whereas the higher frequencies $(3-4 \mathrm{mHz})$ had a relatively high occurrence during more active intervals $\left(K_{\mathrm{p}} \sim 4\right)$. The average Pc 5 power was found to increase significantly with $K_{\mathrm{p}}$, virtually independent of frequency. This means that on a statistical basis, increase geomagnetic activity levels are associated with a broad band enhancement in the Pc 5 amplitude. Frequency shift toward higher frequency with the development of substorm, as observed in cases examined in this study, is consistent with these statistical results. The power increase is dominantly observed in the compressional component in the outer magnetosphere.

Acknowledgments. Thanks are due to the Geotail team at the Institute of Space and Astronautical Science in Japan, for provision of data through DARTS at (http://www.darts.isas.jaxa.jp/stp/geotail/). Geomagnetic data including $\mathrm{AE}$ index were downloaded from the Website of the WDC for Geomagnetism, Kyoto (http://wdc.kugi.kyotou.ac.jp/). Geomagnetic data at Syowa in Antarctic and Tjornes in Iceland were provided through the National Institute of Polar Research (http://polaris.nipr.ac.jp/aurora/). The MACCS array data were available through the Website (http://space.augsburg.edu/space/MaccsHome.html). The solar wind and GOES data were made publicly available through the efforts of the NSSDC at NASA CDAWeb (http://cdaweb. gsfc.nasa.gov/istp_public/).

\section{References}

Anderson, B. J., M. J. Engebretson, S. P. Rounds, L. J. Zanetti, and T. 
A. Potemra, A statistical study of Pc 3-5 pulsations observed by the AMPTE/CCE magnetic fields experiment: 1. Occurrence distributions, J. Geophys. Res., 95, 10,495-10,523, 1990.

Baker, G., E. F. Donovan, and B. J. Jackel, A comprehensive survey of auroral latitude Pc5 pulsationcharacteristics, J. Geophys. Res., 108, 13841397, doi:10.1029/2002JA009801, 2003.

Chen, L. and A. Hasegawa, A theory of long-period magnetic pulsations: 1. Steady state excitation of field line resonances, J. Geophys. Res., 79, 1024-1032, 1974.

Chisham, G. and D. Orr, A statistical study of the local time asymmetry of Pc5 ULF wave characteristics observed at midlatitudes by SAMNET, $J$. Geophys. Res., 102, 24,339-24350, 1997.

Constantinescu, O. D., K.-H. Glassmeier, F. Plaschke, U. Auster, V. Angelopoulos, W. Baumjohann, K.-H. Fornacon, E. Georgescu, D. Larson, W. Magnes, J. P. McFadden, R. Nakamura, and Y. Narita, THEMIS observations of duskside compressional Pc5 waves, J. Geophys. Res., 114, A00C25, doi:10.1029/2008JA013519, 2009.

Engebretson, M. J., W. J. Hughes, J. L. Alford, E. Zesta, L. J. Cahill, Jr., R. L. Arnoldy, and G. D. Reeves, Magnetometer array for cusp and cleft studies observations of the spatial extent of broadband ULF magnetic pulsations at cusp/cleft latitudes, J. Geophys. Res., 100, 19,371-19,386, doi:10.1029/95JA00768, 1995.

Engebretson, M., K.-H. Glassmeier, M. Stellmacher, W. J. Hughes, and $\mathrm{H}$. Lühr, The dependence high-latitude $\mathrm{PcS}$ wave power on solar wind velocity and on the phase of high-speed solar wind streams, J. Geophys. Res., 103, 26,271-26,283, doi:10.1029/97JA03143, 1998.

Fenrich, F. R., J. C. Samson, G. Sofko, and R. A. Greenwald, ULF high and low-m field line resonances observed with the Super Dual Auroral Radar Network, J. Geophys. Res., 100, 21,535-21584, 1995.

Francia, P. and U. Villante, Some evidence of ground power enhancements at frequencies of global magnetospheric modes at low latitude, Ann. Geophys., 15, 17-23, 1997.

Fujita, S., K. H. Glassmeier, and K. Kamide, MHD waves generated by the Kelvin-Helmholtz instability in a nonuniform magnetosphere, $J$. Geophys. Res., 101, 27,317-27,326, 1996.

Hartinger, M., V. Angelopoulos, M. B. Moldwin, Y. Nishimura, D. L. Turner, K.-H. Glassmeier, M. G. Kivelson, J. Matzka, and C. Stolle, Observations of a Pc5 global (cavity/waveguide) mode outside the plasmasphere by THEMIS, J. Geophys. Res., 117, A06202, doi:10.1029/2011JA017266, 2012.

Junginger, H. and W. Baumjohann, Dayside long-period magnetospheric pulsations: Solar wind dependence, J. Geophys. Res., 93, 877-883, doi:10.1029/JA093iA02p00877, 1988.

Junginger, H., G. Haerendel, and F. Melzner, A statistical study of wave Poynting vectors measured during long-period magnetospheric pulsations at geostationary orbit, J. Geophys. Res., 90, 8301-8307, doi:10.1029/JA090iA09p08301, 1985.

Kamide, Y. and S. Kokubun, Two-component auroral electrojet: Importance for substorm studies, J. Geophys. Res., 101, 13,027-13,046, doi:10.1029/96JA00142, 1996.

Kepko, L. and H. E. Spence, Observations of discrete, global magnetospheric oscillations directly driven by solar wind density variations, $J$. Geophys. Res., 108, 1257, doi:10.1029/2002JA009676, 2003.

Kepko, L., H. E. Spence, and H. J. Singer, ULF waves in the solar wind as direct drivers of magnetospheric pulsations, Geophys. Res. Lett., 29, 1197, doi:10.1029/2001GL014405, 2002.

Kikuchi, T., H. Lühr, T. Kitamura, O. Saka, and K. Schlegel, Direct penetration of the polar electric field to the equator during a DP2 event as detected by the auroral and equatorial magnetometer chains and the EISCAT radar, J. Geophys. Res., 101, 17,161-17,173, doi:10.1029/96JA01299, 1996.

Kikuchi, T., H. Luehr, K. Schlegel, H. Tachihara, M. Shinohara, and T.I. Kitamura, Penetration of auroral electric fields to the equator during a substorm, J. Geophys. Res., 105, 23,251-23,261, doi:10.1029/ 2000JA900016, 2000.

Kivelson, M. G. and D. J. Southwood, Resonant ULF waves: A new interpretation, Geophys. Res. Lett., 12, 49-52, 1985.

Kivelson, M. G. and D. J. Southwood, Coupling of global magnetospheric MHD eigenmodes to field line resonances, J. Geophys. Res., 91, 43454351, doi:10.1029/JA091iA04p04345, 1986.

Kokubun, S., Relationship of interplanetary magnetic field structure with development of substorm and storm main phase, Planet. Space Sci., 20, 1033-1049, 1972.

Kokubun, S., Statistical characteristics of Pc 5 waves at geostationary orbit, J. Geomag. Geoelectr., 37, 759-779, 1985.

Kokubun, S. and T. Nagata, Geomagnetic pulsations Pc5 in and near the auroral zones, Rep. Ionos. Space Res. Japan, 19, 158-176, 1965.

Kokubun, S., R. L. McPherron, and C. T. Russell, Ogo 5 observations of Pc 5 waves: Ground magnetosphere correlations, J. Geophys. Res., 81 , 5141-5149, 1976.

Kokubun, S., M. G. Kivelson, R. L. McPherron, C. T. Russell, and H. I. West, Jr., Ogo 5 observations of Pc 5 waves: Particle flux modulations, J. Geophys. Res., 82, 2774-2786, 1977.

Kokubun, S., K. N. Erickson, T. A. Fritz, and R. L. McPherron, Local time asymmetry of Pc 4-5 pulsations and associated particle modulations at synchronous orbit, J. Geophys. Res., 94, 6607-6625, doi:10.1029/JA094iA06p06607, 1989.

Kokubun, S., T. Yamamoto, M. H. Acuna, K. Hayashi, K. Shiokawa, and H. Kawano, The GEOTAIL magnetic field experiment, J. Geomag. Geoelectr., 46, 7-21, 1994.

Kokubun, S., H. Kawano, T. Mukai, Y. Saito, K. Tsuruda, T. Yamamoto, and G. Rostoker, Surface waves on the dawn magnetopause: Connection with ground Pc5 pulsations, Adv. Space Res., 25, 1493-1502, 2000.

Mann, I. R., A. N. Wright, K. J. Mills, and V. M. Nakariakov, Excitation of magnetospheric waveguide modes by magnetosheath flows, J. Geophys. Res., 104, 33-354, 1999.

Mathie, R. A., I. R. Mann, F. W. Menk, and D. Orr, Pc5 ULF pulsations associated with waveguide modes observed with the IMAGE magnetometer array, J. Geophys. Res., 104, 7025-7036, doi:10.1029/1998JA900150, 1999.

Mitchell, D. G., M. J. Engebretson, D. J. Williams, C. A. Catell, and R. Lundin, Pc 5 pulsations in the outer dawn magnetosphere seen by ISEE 1 and 2, J. Geophys. Res., 95, 967-975, 1990.

Miura, A., Kelvin-Helmholtz instability at the magnetospheric boundary: Dependence on the magnetosheath sonic mach number, J. Geophys. Res., 97, 10,655-10,675, doi:10.1029/92JA00791, 1992.

Mukai, T., S. Machida, Y. Saito, M. Hirahara, T. Terasawa, N. Kaya, T. Obara, M. Ejiri, and A. Nishida, The low energy particle (LEP) experiment, on board the GEOTAIL Satellite, J. Geomag. Geoelectr., 46, 669-692, 1994.

Nagata, T., S. Kokubun, and T. Iijima, Geomagnetic conjugate relationships of giant pulsations at Syowa Base, Andtarctica and Reykjavik in Iceland, J. Geophys. Res., 68, 4621-4625, 1963.

Nakamura, M., H. Matsui, H. Kawano, S. Kokubun, K. Takahashi, A. Matsuoka, T. Yamamoto, K. Tsuruda, H. Hayakawa, and T. Okada, Pc 5 pulsations observed in the dayside magnetosphere by Geotail, Geophys. Res. Lett., 21, 2903-2906, 1994.

Nosé, M., T. Iyemori, M. Sugiura, and J. A. Slavin, A strong dawn/dusk asymmetry in Pc5 pulsation occurrence observed by the DE-1 satellite, Geophys. Res. Lett., 22, 2053-2056, doi:10.1029/95GL01794, 1995.

Plaschke, F., K.-H. Glassmeier, H. U. Auster, O. D. Constantinescu, W. Magnes, V. Angelopoulos, D. G. Sibeck, and J. P. McFadden, Standing Alfvén waves at the magnetopause, Geophys. Res. Lett., 36, L02104, doi:10.1029/2008GL036411, 2009a.

Plaschke, F., K.-H. Glassmeier, D. G. Sibeck, H. U. Auster, O. D. Constantinescu, V. Angelopoulos, and W. Magnes, Magnetopause surface oscillation frequencies at different solar wind conditions, Ann. Geophys., 27, 4521-4532, 2009b.

Rostoker, G. and B. Sullivan, Polarization characteristics of Pc5 magnetic pulsations in the dusk hemisphere, Planet. Space Sci., 35, 429-438, 1987.

Ruohoniemi, J. M., R. A. Greenwald, K. B. Baker, and J. C. Samson, HF radar observations of Pc 5 field line resonances in the midnight early morning MLT sector, J. Geophys. Res., 96, 15,697-15710, 1991.

Sakurai, T., Y. Tonegawa, Y. Shinkai, K. Yumoto, S. Kokubun, K. Tsuruda, and T. Mukai, Poynting vectors of Pc 5 pulsations observed by the GEOTAIL satellite in the dayside outer magnetosphere, Earth Planets Space, 53, 843-849, 2001.

Samson, J. C. and G. Rostoker, Latitude-dependent characteristics of highlatitude Pc 4 and Pc 5 micropulsations, J. Geophys. Res., 77, 6133-6144, doi:10.1029/JA077i031p06133, 1972.

Samson, J. C., J. A. Jacobs, and G. Rostoker, Latitude-dependent characteristics of long-period geomagnetic micropulsations, J. Geophys. Res., 76, 3675-3683, doi:10.1029/JA076i016p03675, 1971.

Samson, J. C., R. A. Greenwald, J. M. Ruohoniemi, T. J. Hughes, and D. D. Wallis, Magnetometer and radar observations of magnetospheric cavity modes in the Earth's magnetosphere, Can. J. Phys., 69, 929-937, 1991.

Samson, J. C., D. D. Wallis, T. J. Hughes, F. Creutzberg, J. M. Ruohoniemi, and R. A. Greenwald, Substorm intensifications and field line resonances in the nightside magnetosphere, J. Geophys. Res., 97, 8495, $1992 \mathrm{a}$.

Samson, J. C., B. G. Harrold, J. M. Ruohoniemi, R. A. Greenwald, and A. 
D. M. Walker, Field line resonances associated with MHD waveguides in the magnetosphere, Geophys. Res. Lett., 19, 441-444, 1992b.

Shimazu, H., T. Araki, T. Kamei, and H. Hanado, A symmetric appearance of Pc5 on dawn and dusk side associated with solar wind dynamic pressure enhancement, J. Geomag. Geoelectr., 47, 177-189, 1995.

Singer, H. J. and M. G. Kivelson, The latitudinal structure of Pc5 waves in space: Magnetic and electric observations, J. Geophys. Res., 84, 72137222, doi:10.1029/JA084iA12p07213, 1979.

Singer, H. J., W. J. Hughes, and C. T. Russell, Standing hydromagnetic waves observed by ISEE 1 and 2: Radial extent and harmonic, J. Geophys. Res., 87, 3519-3529, 1982.

Southwood, D. J., Some features of field line resonances in the magnetosphere, Planet Space Sci., 22, 483-491, 1974.

Southwood, D. J., A general approach to low-frequency instability in the ring current plasma, J. Geophys. Res., 81, 3340-3348, 1976.

Tamao, T., Transmission and coupling resonance of hydromagnetic disturbances in the non-uniform Earth's magnetosphere, Sci. Rep. Tohoku Univ. Geophys., 17, 43-72, 1965.

Tsuruda, K., H. Hayakawa, M. Nakamura, T. Okada, M. Matsuoka, F. S. Mozer, and R. Schmidt, Electric field measurements on the GEOTAIL Satellite, J. Geomag. Geoelectr., 46, 693-712, 1994.

Vennerstrøm, S., Dayside magnetic ULF power at high latitudes: A possible long-term proxy for the solar wind velocity, J. Geophys. Res., 104, 10,145, 1999.

Viall, N. M., L. Kepko, and H. E. Spence, Relative occurrence rates and connection of discrete frequency oscillations in the solar wind density and dayside magnetosphere, J. Geophys. Res., 114, A01201, doi:10.1029/2008JA013334, 2009.

Villante, U., P. Francia, and S. Lepidi, Pc5 geomagnetic field fluctuations at discrete frequencies at a low latitude station, Ann. Geophys., 19, 321325, 2001.

Villante, U., M. Vellante, P. Francia, M. De Lauretis, A. Meloni, P. Palangio, B. Zolesi, M. Pezzopane, M. Forster, T. L. Zhang, W. Magnes, P. Nenovski, I. Cholakov, and V.Wesztergom, ULF fluctuations of the geomagnetic field and ionospheric sounding measurements at low latitudes during the first CAWSES campaign, Ann. Geophys., 24, 1455-1468, 2006.

Villante, U., P. Francia, M. Vellante, P. Di Giuseppe, A. Nubile, and M. Piersanti, Long-period oscillations at discrete frequencies: A comparative analysis of ground, magnetospheric, and interplanetary observations, J. Geophys. Res., 112, A04210, doi:10.1029/2006JA011896, 2007.

Walker, A. D. M., J. M. Ruohoniemi, K. B. Baker, R. A. Greenwald, and J. C. Samson, Spatial and temporal behavior of ULF pulsations observed by the Goose Bay HF radar, J. Geophys. Res., 97, 12,187-12,202, 1992.

Zhu, X. and M. G. Kivelson, Compressional ULF waves in the outer magnetosphere, 1. Statisticalstudy, J. Geophys. Res., 96, 19,451-194657, 1991.

Ziesolleck, C. W. S. and D. R. McDiarmid, Auroral latitude Pc 5 field line resonances: Quantized frequencies, spatial characteristics, and diurnal variations, J. Geophys. Res., 99, 5817-5830, 1994.

Ziesolleck, C. W. S. and D. R. McDiarmid, Statistical survey of auroral latitude Pc 5 spectral and polarization characteristics, J. Geophys. Res., 100, 19,299-19,312, 1995.

S. Kokubun (e-mail: kokubun@pop21.odn.ne.jp) 